In print in Studia Logica. Post-print.

\title{
Adaptive Fregean Set Theory
}

\author{
Diderik Batens* \\ Centre for Logic and Philosophy of Science \\ Universiteit Gent, Belgium \\ Diderik.Batens@ugent.be
}

\begin{abstract}
This paper defines provably non-trivial theories that characterize Frege's notion of a set, taking into account that the notion is inconsistent. By choosing an adaptive underlying logic, consistent sets behave classically notwithstanding the presence of inconsistent sets. Some of the theories have a full-blown presumably consistent set theory $T$ as a subtheory, provided $T$ is indeed consistent. An unexpected feature is the presence of classical negation within the language.
\end{abstract}

Keywords: Fregean set theories, adaptive logics, content guidance, paraconsistency.

\section{Aim Of This Paper}

This paper concerns non-trivial theories that characterize Frege's notion of a set. There are several such theories and they have remarkable properties.

While the first proposed paraconsistent set theory [11] proceeded in the wake of Quine's NF, a lot of attention was paid to paraconsistent versions of Frege's set theory $[10,17,27]$. The topic is fascinating for several reasons. First, Frege's notion of a set is generally considered natural, as is witnessed by the many mathematics curricula that offer a course on naive set theory. Next, it is important that we try to understand the way in which mathematicians moved from Frege's inconsistent set theory to the presumably consistent set theories that succeeded it.

The third reason requires a few more words. While many believe that the restrictions imposed by the $\mathbf{Z F}$-axioms are sufficient to avoid the antinomies, doubts were raised, from the outset, on the issue whether those restrictions are necessary. Meanwhile paraconsistent logics came into being and gained recognition. As a result, a more general question may be raised: Is it possible

\footnotetext{
* Part of the ideas and results were presented in conferences. My gratitude goes (i) to the audience of the 5th World Congress on Universal Logic (Istanbul, Turkey, 20-30 June 2015) for questions and suggestions, especially to Graham Priest with whom I also corresponded on the matter afterwards; (ii) to the attentive and helpful audience of an $\exists$ ntia et Nomin $\forall$ Workshop (Krakow, Poland, 9-11 September 2015); and (iii) to both referees for pointing out ways to improve the paper.
} 
to devise an inconsistent but non-trivial set theory that is based on Frege's notion but nevertheless serves the purposes a decent set theory should serve?

Some specifications are required. First, I do not aim at a set theory that Frege would have liked; as I understand Frege, he would have considered an inconsistent set theory unpalatable. I am after a set theory "based on Frege's notion", viz. with 'the same' axioms as Frege's. There is a complication because classical logic $\mathbf{C L}$ is unavoidably replaced by a paraconsistent logic and because Frege's axioms, even some instances of the abstraction axiom schema Abs by themselves, are CL-trivial. Here is how to get around that. Two strings $\sigma_{1} \sigma_{2} \ldots \sigma_{n}$ and $\tau_{1} \tau_{2} \ldots \tau_{n}$ are symbol-wise equivalent in $\mathbf{C L}$ iff (i) $\sigma_{i}=\tau_{i}$ if $\sigma_{i}$ is not a logical symbol and (ii) $\sigma_{i}$ and $\tau_{i}$ have the same meaning within $\mathbf{C L}$ if $\sigma_{i}$ is a logical symbol. ${ }^{1}$ Desideratum 1: every axiom of the paraconsistent set theory is symbol-wise equivalent to one of Frege's axioms and vice versa.

It seems desirable to accompany the formal Desideratum 1 with some substantive demands. First, sets should be truly extensional. Desideratum 2: two sets are identical just in case they have the same members as well as the same non-members. Next, Abstraction should be taken serious. Desideratum 3: to every property corresponds a set such that (i) every object that has (does not have) the property is a member (non-member) of the set and (ii) no object is a member (non-member) of a set without a reason.

While inconsistencies are unavoidable, we want the paraconsistent set theory to be consistent where it can be consistent. We should tread carefully here. If it is unavoidable that one of three sets is inconsistent and no further information is available, then there is no systematic formal reason to select any of the three as consistent. This leads to Desideratum 4: if a set (or sentence) can be selected as consistent in view of a systematic formal reason, then the set (or sentence) is consistent.

To serve the purposes a decent set theory should serve, it seems to me that the inconsistent set theory should include a consistent set theory. In view of the generality of the Abs schema, that does not even sound difficult. The consistent theory may require a restricted membership relation - the universal set is not a member of any set in ZF. As I shall be unable to say much on the topic in this paper, I phrase a rather vague Desideratum 5: if there are consistent set theories, they are subtheories of the paraconsistent Fregean set theory.

The main aim of this paper is to devise set theories that fulfil the five desiderata as well as obvious desiderata, for example that I am after a non-trivial theory in the set theoretic language.

The aforementioned publications on paraconsistent set theory were all impressive at the time of their appearance. They are, however, not satisfactory with respect to the five desiderata. Set theories that have no detachable implication fail with respect to desiderata 2,3,4 and 5. Even $\emptyset \in\{\emptyset\}$ and $\{x \mid x \notin x\} \notin\{\emptyset\}$ are not provable if the theories meet Desideratum 1; actually nearly nothing is. ${ }^{2}$ Set theories phrased in terms of a relevant implication turn identity into an intensional operator; all those known to me fail Desideratum 2.

\footnotetext{
${ }^{1}$ The paraconsistent logic CLuNs, presented in Section 2, has, for example, implications that have different meanings within CLuNs but the same meaning within CL.

${ }^{2}$ More is provable if one defines $t_{1}=t_{2}={ }_{d f} \forall x\left(x \in t_{1} \Leftrightarrow x \in t_{2}\right)$, in which $A \Leftrightarrow B={ }_{d f}$ $(\neg A \vee B) \wedge(A \vee \neg B)$. Yet, the defined identity is non-transitive: in some models $M$ there is a set $t_{1}$ such that $M \Vdash \forall x\left(x \in t_{1} \wedge x \notin t_{1}\right)$ as well as at least two consistent sets, $t_{2}$ and $t_{3}$, that differ from each other. So $M \Vdash \forall x x=t_{1}$ and $M \nVdash t_{2}=t_{3}$.
} 
That $\{x \mid A(x)\}$ and $\{x \mid B(x)\}$ have the same members as well as the same non-members is insufficient for proving $\{x \mid A(x)\}=\{x \mid B(x)\}$; this requires a proof of $\forall x(A(x) \leftrightarrow B(x))$ for $\leftrightarrow$ the relevant equivalence of the set theory. ${ }^{3}$

Where $R$ names the Russell set, $\{x \mid \neg x \in x\}$, the paraconsistent Fregean set theory we are after will unavoidably have $R \in R \wedge \neg R \in R$ as a theorem. So there are inconsistent sets, sets of which some members are also non-members, next to consistent sets; and the negation $\neg$ has to be paraconsistent. Yet, we want to interpret the inconsistent theory as consistently as possible. As $\neg R \in R$ and $R \in R \vee A$ hold for all $A$, to derive $A$ from these formulas by Disjunctive Syllogism is simply an inferential mistake. Yet if $t_{1}$ is a consistent set, and even if $t_{2}$ is a non-member of $t_{1}$ and not also a member of $t_{1}, A$ is true whenever $\neg t_{2} \in t_{1}$ and $t_{2} \in t_{1} \vee A$ are true. Capitalizing on consistent membership is the more justified as Frege (mistakenly) believed and (for good reasons) intended his theory to be consistent.

An inconsistency-adaptive approach seems appropriate. Details follow in Section 2, but here are some suggestions. Inconsistency-adaptive logics allow for inconsistencies and possibly for other abnormalities, but localize and minimize them. Parts of the theory that do not involve an abnormality behave as they would in terms of CL. Actually, the type of problem under consideration is the main intended application context of inconsistency-adaptive logics: to obtain a minimally inconsistent 'interpretation' of theories that were intended as consistent but turned out inconsistent. However, the original intention was to use the minimally inconsistent 'interpretation' as a starting point to devise a consistent theory. Only much later came the idea to devise theories the underlying logic of which is adaptive. Such theories are not semi-recursive sets of formulas, but are up to $\Pi_{1}^{1}$-complex. In view of Desideratum 4 , no simpler theory will do for theories that explicate the Fregean notion of a set.

Joint work with Peter Verdée led to a lecture at the 2006 Studia Logica Conference in Torun. Verdée continued to work on the topic. This resulted in several lectures and papers [24, 25] about some four Fregean set theories. For all straightforward choices of adaptive logics $A L$, Verdée found that the presence of Curry sets caused a major problem. The gain expected from the adaptive approach was not obtained. So the adaptive set-theories failed Desideratum 4. Verdée was able to devise adaptive set theories that, by present lights, fulfil Desideratum 4. Unfortunately, each of these fails Desideratum 1; they either add an axiom or impose a restriction on an axiom. Other proposals are ad hoc in requiring an adaptive logic that is not in standard format.

This first paper on the topic proceeds in terms of Abstraction rather than (full) Comprehension - see Section 4. The paper may be rewritten in terms of Comprehension, but I avoid connected difficulties for now.

One of the extremely impressive properties of the adaptive Fregean set theories AFS is that the classical negation is locally definable. This will obviously be explained, but what it comes to is that a classical negation may be introduced from the very beginning, thus impressively raising the expressive power of the AFS. For example, notwithstanding the presence of a non-triviality proof, the

\footnotetext{
${ }^{3}$ Zach Weber [26] studied so-called Doppelgänger, sets that have the same members without being identical. He shows, among other things, that the considered examples should not be identical because they differ with respect to non-membership. Yet, he does not even mention sets for which $\forall x(A(x) \leftrightarrow B(x))$ is unprovable even if $\{x \mid A(x)\}$ and $\{x \mid B(x)\}$ have the same members as well as the same non-members.
} 
'classical' Russell set exists - a drastic difference with all paraconsistent set theories known to me. Other impressive features are saved for Section 9 because stating them here would require too lengthy an explanation.

In view of space limitations, the present paper does not contain all available results. It describes an approach that leads to adaptive Fregean set theories that fulfil the desiderata. The results show the approach sensible and promising. Needless to say, the results do not circumvent the limitative theorems or produce other fake miracles. That they offer perspectives that differ from the traditional ones is fascinating enough.

\section{Logical and Linguistic Preliminaries}

In this paper, the metalanguage will be classical and "false" will function as the classical negation of "true". So nothing will be said to be both true and false, but some formulas will be said to be true together with their negation. I shall need set-theoretic statements in the metalanguage and these will be classical, say ZFC-ish. Confusion is excluded in that all metalinguistic statements will contain symbols that do not belong to the object language.

A metalinguistic expression of the form $\mathrm{A}(\alpha)$ will denote a formula in which at most the variable $\alpha$ occurs free. Let $\exists A$, respectively $\forall A$, denote $A$ preceded by an existential, respectively universal, quantifier over every variable free in $A$; let $\mathrm{Q} A$ denote $A$ preceded by a quantifier over every variable free in $A$.

Let $\mathcal{L}_{s}$ be the standard predicative language schema with the following logical symbols $\{\neg, \wedge, \vee, \supset, \equiv, \forall, \exists,=\}$. The sets of sentential letters, individual constants, individual variables, and predicates of $\operatorname{rank} r$ will be named $\mathcal{S}, \mathcal{C}, \mathcal{V}$, and $\mathcal{P}^{r}$ respectively. Let $\mathcal{F}_{s}$ be the set of formulas of $\mathcal{L}_{s}$ and $\mathcal{W}_{s}$ be the set of closed formulas of $\mathcal{L}_{s}$

The popular paraconsistent logic CLuNs is known under many names. I refer to a surveying study [8] for further references. The version of CLuNs that I shall need consists of full positive CL including Replacement of Identicals (RoI), $\forall x \forall y(x=y \supset(A(x) \supset A(y)))$, Excluded Middle, $A \vee \neg A$, plus the standard axioms to drive negation inwards: $\neg \neg A \equiv A, \neg(A \wedge B) \equiv \neg A \vee \neg B$, $\ldots, \neg \exists x A \equiv \forall x \neg A$.

The handiest CLuNs-semantics for present purposes is the three-valued one, with values $T$ (consistently true), $I$ (inconsistent), and $F$ (false). By $\mathcal{L}_{\mathcal{O}}$ I shall mean a pseudo-language schema that is exactly like the standard predicative language schema $\mathcal{L}_{s}$, except that the role of the countable set $\mathcal{C}$ of individual constants is played by $\mathcal{C} \cup \mathcal{O}$, with $\mathcal{O}$ a possibly uncountable set of pseudoconstants. ${ }^{4}$ Let $\mathcal{W}_{\mathcal{O}}$ be the set of closed pseudo-formulas of $\mathcal{L}_{\mathcal{O}}$. In the CLuNssemantics, each model $M=\langle\mathrm{D}, v\rangle$ is described in terms of an $\mathcal{L}_{\mathcal{O}}$ in which $\mathcal{C} \cup \mathcal{O}$ has at least the cardinality of D. ${ }^{5}$

Where $M=\langle\mathrm{D}, v\rangle$ is a CLuNs-model, $\mathrm{D}$ is a set and $v$ an assignment function defined as follows. (i) Sentential letters are assigned a truth value, $v: \mathcal{S} \rightarrow\{T, I, F\}$. (ii) Constants and pseudo-constants are mapped to the

\footnotetext{
${ }^{4}$ The standard view requires (languages and) language schemes to have countably many symbols. The prefix "pseudo-" indicates that countability may fail.

${ }^{5}$ Phrasing the semantics in terms of $\mathcal{L}_{\mathcal{O}}$ is just a neater way to phrase the semantics as if "the elements of the domain D were names for themselves" (which strictly speaking is nonsense). Doing as if one has a name for every element of D is fictional (for uncountable D), but allows for a simpler handling of the quantifiers than less fictional descriptions.
} 
domain, $v: \mathcal{C} \cup \mathcal{O} \rightarrow \mathrm{D}$, in such a way that $\{v(\alpha) \mid \alpha \in \mathcal{C} \cup \mathcal{O}\}=\mathrm{D}$. (iii) Predicates of rank $r$ are assigned a triple of $r$-tuples of $\mathrm{D},\left\langle\Sigma_{1}, \Sigma_{2}, \Sigma_{3}\right\rangle$, such that $\Sigma_{1} \cup \Sigma_{2} \cup \Sigma_{3}=\wp\left(\mathrm{D}^{r}\right)$ and $\Sigma_{1} \cap \Sigma_{2}=\Sigma_{1} \cap \Sigma_{3}=\Sigma_{2} \cap \Sigma_{3}=\emptyset$. To simplify the notation, consider $v$ as composed in this case of the three functions $v^{T}, v^{I}$, and $v^{F}$, with $v^{T}\left(\pi^{r}\right)=\Sigma_{1}, v^{I}\left(\pi^{r}\right)=\Sigma_{2}$, and $v^{F}\left(\pi^{r}\right)=\Sigma_{3}$. Identity is handled as a binary predicate with the special restriction that $v^{T}(=) \cup v^{I}(=)=\{\langle o, o\rangle \mid o \in \mathrm{D}\}$.

The valuation determined by $M, v_{M}: \mathcal{W}_{\mathcal{O}} \rightarrow\{T, I, F\}$, handles atomic formulas as follows: ${ }^{6}$

$$
\begin{aligned}
& \text { where } A \in \mathcal{S}, v_{M}(A)=v(A) \\
& v_{M}\left(\pi^{r} \alpha_{1} \ldots \alpha_{r}\right)=T \text { iff }\left\langle v\left(\alpha_{1}\right), \ldots, v\left(\alpha_{r}\right)\right\rangle \in v^{T}\left(\pi^{r}\right) \\
& v_{M}\left(\pi^{r} \alpha_{1} \ldots \alpha_{r}\right)=I \text { iff }\left\langle v\left(\alpha_{1}\right), \ldots, v\left(\alpha_{r}\right)\right\rangle \in v^{I}\left(\pi^{r}\right) \\
& v_{M}\left(\pi^{r} \alpha_{1} \ldots \alpha_{r}\right)=F \text { iff }\left\langle v\left(\alpha_{1}\right), \ldots, v\left(\alpha_{r}\right)\right\rangle \in v^{F}\left(\pi^{r}\right)
\end{aligned}
$$

\begin{tabular}{|c|c|c|c|c|c|c|c|c|c|}
\hline & $\neg$ & $\supset$ & $T$ & $I$ & $F$ & $\wedge$ & $T$ & $I$ & $F$ \\
\hline $\bar{T}$ & $F$ & $T$ & $T$ & $I$ & $\bar{F}$ & $T$ & $T$ & $I$ & $F$ \\
\hline$I$ & $I$ & $I$ & $T$ & $I$ & $F$ & $I$ & $I$ & $I$ & $F$ \\
\hline$F$ & $T$ & $F$ & $T$ & $T$ & $T$ & $F$ & $F$ & $F$ & $F$ \\
\hline
\end{tabular}

The meaning of three connectives is defined by the matrices

and the value of universally quantified formulas is determined by

$$
\begin{aligned}
& v_{M}(\forall \alpha A(\alpha))=T \text { iff } v_{M}(A(\beta))=T \text { for all } \beta \in \mathcal{C} \cup \mathcal{O} \\
& v_{M}(\forall \alpha A(\alpha))=F \text { iff } v_{M}(A(\beta))=F \text { for at least one } \beta \in \mathcal{C} \cup \mathcal{O} \\
& v_{M}(\forall \alpha A(\alpha))=I \text { otherwise. }^{7}
\end{aligned}
$$

The remaining logical symbols are defined explicitly:

$$
\begin{aligned}
& A \vee B={ }_{d f} \neg(\neg A \wedge \neg B) \\
& A \equiv B={ }_{d f}(A \supset B) \wedge(B \supset A) \\
& \exists \alpha A(\alpha)={ }_{d f} \neg \forall \alpha \neg A(\alpha)
\end{aligned}
$$

$M \Vdash A$ ( $M$ verifies $A$ ) iff $v_{M}(A) \in\{T, I\}$. $M$ is a model of $\Gamma$ iff $M \Vdash A$ for all $A \in \Gamma . \Gamma \vDash A$ ( $A$ is a semantic consequence of $\Gamma$ ) iff all models of $\Gamma$ verify $A . \vDash A(A$ is valid $)$ iff all models verify $A .^{8}$ Where $T=\langle\Gamma, \mathbf{L}\rangle$ is a theory, with $\Gamma$ a set of non-logical axioms and $\mathbf{L}$ a logic, $\vdash_{\mathbf{T}} A$ abbreviates $\Gamma \vdash_{\mathbf{L}} A$.

The following statements give a feel of detachment in CLuNs:

$$
\begin{aligned}
& A \supset B, A \vdash_{\mathbf{C L u N s}} B \\
& A \supset B, \neg B \nvdash_{\mathrm{CLuNs}} \neg A \quad \text { but } \quad A \supset B, \neg B \vdash_{\mathrm{CLuNs}} \neg A \vee(B \wedge \neg B) \\
& A \vee B, \neg B \nvdash_{\mathrm{CLuNs}} A \text { but } A \vee B, \neg B \vdash_{\mathrm{CLuNs}} A \vee(B \wedge \neg B)
\end{aligned}
$$

Many further logical symbols are definable within CLuNs. Thus $A \sqsupset B=d f$ $\neg A \vee B$ is contraposable but not detachable, $A \rightarrow B=_{d f}(A \supset B) \wedge(\neg B \supset \neg A)$ is contraposable and detachable. Apart from the earlier introduced $\Leftrightarrow$, two definable equivalences will play a role in the sequel:

$$
\begin{array}{cc}
A \leftrightarrow B \quad=_{d f} & (A \equiv B) \wedge(\neg A \equiv \neg B) \\
A \Leftrightarrow B \quad=_{d f} & (B \supset A) \wedge(\neg B \supset \neg A)
\end{array}
$$

\footnotetext{
${ }^{6}$ The aim is a semantics for $\mathcal{L}_{s}$ and $\mathcal{W}_{s} \subseteq \mathcal{W}_{\mathcal{O}}$.

${ }^{7}$ Viz. iff $v_{M}(A(\beta)) \in\{T, I\}$ for all $\beta \in \mathcal{C} \cup \mathcal{O}$ and $v_{M}(A(\beta))=I$ for at least one $\beta \in \mathcal{C} \cup \mathcal{O}$

${ }^{8}$ Propositional CLuNs is decidable. Drive negations inward and next proceed as for propositional CL, except that both $A$ and $\neg A$ may be true. In view of this, I shall leave it to the reader to spell out propositional steps (as well as obvious steps from positive logic).
} 
Both $(A \leftrightarrow B) \leftrightarrow(B \leftrightarrow A)$ and $(A \leftrightarrow B) \leftrightarrow(\neg B \leftrightarrow \neg A)$ are CLuNstheorems, $\leftrightarrow$ is detachable and Replacement of Equivalents holds for it. The asymmetric equivalence $\Longleftrightarrow$ has a strong right-left direction and a weak left-right direction: $A \Longleftrightarrow B$ CLuNs-entails $B \supset A$ and $\neg B \supset \neg A$, but only $A \sqsupset B$ and $\neg A \sqsupset \neg B$. While $(A \Leftrightarrow B) \leftrightarrow(\neg A \Longleftrightarrow \neg B)$ is a CLuNs-theorem, neither $(A \Longleftrightarrow B) \leftrightarrow(B \Longleftrightarrow A)$ nor $(A \Longleftrightarrow B) \leftrightarrow(\neg B \Longleftrightarrow \neg A)$ is.

Frege's axioms Ext and Abs will be introduced in Section 4. The theory defined from these axioms by CLuNs will be called PFS (Paraconsistent Fregean Set theory). PFS is not the theory I am after but only a preparatory step towards Adaptive Fregean Set theories AFS. As there is a manifold of them and many are interesting, AFS does not name a system but the family of systems. All members of the family are defined from the two PFS-axioms by different adaptive logics 'based on' CLuNs.

Several introductions to (inconsistency-)adaptive logics are available, including recent ones $[3,7,6,20]$. So I shall stick to the bare essentials.

An adaptive logic is a triple composed of (i) a lower limit logic, which is a logic that has static proofs [4] - roughly a Tarski logic-(ii) a set of abnormalities $\Omega$, which is a subset of $\mathcal{W}_{s}$ characterized by a possibly restricted logical form, and (iii) an adaptive strategy. In the present paper, we shall only consider adaptive logics that have CLuNs as their lower limit and Minimal Abnormality as their strategy. By way of example, let $\Omega=\left\{\mathrm{Q}(A \wedge \neg A) \mid A \in \mathcal{F}^{a}\right\}$, in which $\mathcal{F}^{a}$ is the set of atomic formulas (those that contain no logical symbols other than identity). ${ }^{9}$ The logic determined by these choices, will be called $\mathbf{C L u N s}{ }^{m} .{ }^{10}$ The underlying logic of the AFS that will be introduced later will be different from $\mathbf{C L u N s}^{m}$. Yet, the latter is a good starting point to explain the idea.

Like all adaptive logics that have Minimal Abnormality as their strategy, $\mathbf{C L u N s}^{m}$ is most easily understood in semantic terms. Where $M$ is a CLuNsmodel, $A b(M)=\{A \in \Omega \mid M \Vdash A\} . M$ is a minimally abnormal CLuNs-model of $\Gamma$ iff $M$ is a CLuNs-model of $\Gamma$ and no CLuNs-model $M^{\prime}$ of $\Gamma$ is such that $A b\left(M^{\prime}\right) \subset A b(M) . \Gamma \vDash_{\mathbf{C L u N s}^{m}} A$ iff every minimally abnormal CLuNs-model of $\Gamma$ verifies $A$.

The most fascinating aspect of adaptive logics are the dynamic proofs. These offer a syntactic definition of $\mathbf{C L u N s}{ }^{m}$-consequence sets and at the same time explicate the way in which we estimate membership of such a consequence set by finitary means.

Lines of annotated dynamic proofs consist of four elements: a number, a formula, a justification, and a condition, which is a finite subset of $\Omega$. Where (i) $\Gamma$ is the premise set, (ii) $\operatorname{Dab}(\Delta)$ denotes a disjunction of the members of a finite $\Delta \subseteq \Omega$, and (iii)

\section{$A \quad \Delta$}

abbreviates a line at which $A$ occurs on the condition $\Delta$, the rules of inference can be phrased as three generic rules:

\footnotetext{
${ }^{9}$ Extending the lower limit logic CLuNs with an axiom that abnormalities entail triviality- $(A \wedge \neg A) \supset B$ will do-delivers the upper limit logic, in the present case CL.

${ }^{10}$ In many papers, CLuNs ${ }^{m}$ names a variant with $\Omega=\left\{\exists(A \wedge \neg A) \mid A \in \mathcal{F}^{a}\right\}$.
} 
Prem If $A \in \Gamma$ :

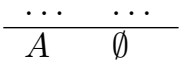

RU

If $A_{1}, \ldots, A_{n} \vdash_{\text {CluNs }} B$ :

$A_{1} \quad \Delta_{1}$

... $\quad \cdots$

\begin{tabular}{ll}
$A_{n}$ & $\Delta_{n}$ \\
\hline$B$ & $\Delta_{1} \cup \ldots \cup \Delta_{n}$
\end{tabular}

$\mathrm{RC}$

$$
\begin{aligned}
& \text { If } A_{1}, \ldots, A_{n} \vdash_{\text {CLuNs }} B \vee \operatorname{Dab}(\Theta): \quad A_{1} \quad \Delta_{1} \\
& \text {... } \quad \ldots \\
& \begin{array}{ll}
A_{n} & \Delta_{n} \\
\hline B & \Delta_{1} \cup \ldots \cup \Delta_{n} \cup \Theta
\end{array}
\end{aligned}
$$

Only RC introduces new non-empty conditions. Prem introduces empty conditions and RU merely adds up conditions into a union. ${ }^{11}$ Note that CLuNs is a compact logic and that the above generic rules are finitary, viz. have a finite number of local premises. A proof stage is a sequence of lines. A proof is a chain of stages. An application of a rule brings the proof to the next stage.

At each stage $s$, the marking definition determines for every line $i$ whether $i$ is unmarked or marked - this means that it is respectively IN or OUT - in view of (i) the condition of $i$ and (ii) the minimal $D a b$-formulas of stage $s$. I shall only consider the marking definition for Minimal Abnormality. A choice set of $\Sigma=\left\{\Delta_{1}, \Delta_{2}, \ldots\right\}$ is a set that contains an element out of each member of $\Sigma$. A minimal choice set of $\Sigma$ is a choice set of $\Sigma$ of which no proper subset is a choice set of $\Sigma$. Where $\operatorname{Dab}\left(\Delta_{1}\right), \ldots, \operatorname{Dab}\left(\Delta_{n}\right)$ are the $D a b$-formulas that occur on the empty condition in stage $s, \Phi_{s}(\Gamma)$ is the set of the minimal choice sets of $\left\{\Delta_{1}, \ldots, \Delta_{n}\right\}$. Let $\operatorname{Dab}\left(\Delta_{1}\right), \operatorname{Dab}\left(\Delta_{2}\right), \ldots$ be the $D a b$-formulas that are CLuNs-derivable from $\Gamma$ and let $\Phi(\Gamma)$ be the set of minimal choice sets of $\Sigma=\left\{\Delta_{1}, \Delta_{2}, \ldots\right\}$. It was proved [3, Lemma 4] that $\varphi \in \Phi(\Gamma)$ iff there is a Minimal Abnormal CLuNs-model $M$ of $\Gamma$ such that $\varphi=A b(M) . \Phi_{s}(\Gamma)$ may be seen as the best estimate, at stage $s$, of $\Phi(\Gamma)$.

Definition 1 Marking for Minimal Abnormality: where $A$ is the formula and $\Delta$ is the condition of line $i$, line $i$ is marked at stage $s$ iff (i) there is no $\varphi \in \Phi_{s}(\Gamma)$ such that $\varphi \cap \Delta=\emptyset$, or (ii) for some $\varphi \in \Phi_{s}(\Gamma)$, there is no line at which $A$ is derived on a condition $\Theta$ for which $\varphi \cap \Theta=\emptyset$.

Definition $2 A$ is finally derived from $\Gamma$ at line $i$ of a finite $\mathbf{C L u N s}^{m}$-proof stage $s$ iff (i) $A$ is the second element of line $i$, (ii) line $i$ is not marked at stage $s$, and (iii) every extension of the proof in which line $i$ is marked may be further extended in such a way that line $i$ is unmarked.

Definition $3 \Gamma \vdash_{\mathbf{C L u N s}^{m}} A$ ( $A$ is finally $\mathbf{C L u N s}^{m}$-derivable from $\Gamma$ ) iff $A$ is finally derived at a line of a $\mathbf{C L u N s}{ }^{m}$-proof stage from $\Gamma$.

Marks at stage $s$ may be seen as the estimate of final derivability in view of insights available at $s$. Establishing final $\mathbf{C L u N s}{ }^{m}$-derivability requires (i) a finite proof stage and (ii) a metatheoretic reasoning about extensions of the stage

\footnotetext{
${ }^{11}$ Provably [3, Lemma 1] a line at which $A$ occurs on the condition $\Delta$ can be obtained in a CLuNs $^{m}$-proof from $\Gamma$ iff $\Gamma \vdash_{\text {CLuNs }} A \vee D a b(\Delta)$; the latter obtains iff, in a CLuNs-proof from $\Gamma$, a line can be obtained at which $A \vee \operatorname{Dab}(\Delta)$ is derived on the condition $\emptyset$.
} 
and extensions of these. I shall consider other adaptive logics, all of which will have CLuNs as their lower limit logic. Semantic consequence and derivability for those logics are as for $\mathbf{C L u N s}^{m}$ - only $\Omega$ and the name of the adaptive logic need to be replaced. For all of them holds $\Gamma \vdash A$ iff $\Gamma \vDash A$ [3, Theorem 9].

In AFS, abnormalities are allowed but minimized. In semantic terms, the models of an AFS are a selection of the PFS-models. So AFS have all PFStheorems as theorems. Interesting AFS have further theorems; these form the adaptive gain.

The reason to choose CLuNs rather than another paraconsistent logic as underlying PFS is mainly a matter of experience. In comparison to empirical theories, mathematical theories are conceptually simple, they are not blurred by empirical data, and their conceptual difficulties relate to properties of their most atomic concepts. CLuNs connects inconsistencies in that removing some of them comes to removing infinitely many of them at once. ${ }^{12}$ In mathematical theories, disconnecting those inconsistencies by moving to a weaker logic does not open any interesting roads for removing involved inconsistencies.

The choice for $\mathbf{C L u N s}^{m}$ is provisional but, at this point, natural. We shall see that PFS requires some atomic contradictions to be true, for example $R \in$ $R \wedge R \notin R$, in which $R$ is the Russell set. The natural reaction is to allow for contradictions but to minimize them. Minimizing contradictions in CLuNs has to be done by minimizing atomic contradictions - otherwise a flip-flop results, as was shown a long time ago $\left[2\right.$, p. 50]. ${ }^{13}$

\section{The Language of Inconsistent Set Theory}

The language of set theory, $\mathcal{L}_{S}$, takes part of its signature from $\mathcal{L}_{s}$, viz. $\{\neg$, $\wedge, \vee, \supset, \equiv, \forall, \exists,=\} \cup \mathcal{V}$. Apart from these, there is the binary predicate $\in$ and there are abstracting terms $\{\alpha \mid A(\alpha)\}$, in which $A(\alpha)$ is called the touchstone of the abstracting term - I shall sometimes write "set" instead of "abstracting term". We stipulate that $\alpha$ is bound in $\{\alpha \mid A(\alpha)\}$. The only terms are abstracting terms and members of $\mathcal{V}$; all individual constants will be abbreviations of abstracting terms. Let $\mathcal{T}$ be the set of terms and $\mathcal{T}^{c}$ the set of constant terms. Let $\mathcal{F}_{S}$ be the set of formulas of $\mathcal{L}_{S}$ and $\mathcal{W}_{S}$ be the set of sentences (closed formulas) of $\mathcal{L}_{S}$.

$$
\begin{array}{rlrl}
!(A) & { }_{d f} & A \wedge \neg A \\
t_{1} \neq t_{2} & ={ }_{d f} & \neg t_{1}=t_{2} \\
t_{1} \notin t_{2} & ={ }_{d f} & \neg t_{1} \in t_{2} \\
\perp & ={ }_{d f} & \forall x \forall y(x=y \wedge x \neq y \wedge x \in y \wedge x \notin y) \\
\top & ={ }_{d f} & \perp \supset \perp \\
\sim A & { }_{d f} & A \supset \perp
\end{array}
$$

\footnotetext{
${ }^{12}$ Where $M$ is a CLuNs-model, (i) $M \Vdash A \wedge \neg A$ iff $M \Vdash(A \wedge \neg A) \wedge \neg(A \wedge \neg A)$, (ii) $M \Vdash A \wedge \neg A$ iff $M \Vdash(A \wedge B) \wedge \neg(A \wedge B)$ whenever $M \Vdash B$, and so on.

${ }^{13}$ A flip-flop delivers the upper limit consequence set of normal premise sets, as do all adaptive logics, but delivers only the lower limit consequences of inconsistent premise sets. So there is no adaptive gain if the premise set requires some abnormalities to be true.
} 
Clearly $T$ is a CLuNs-theorem and in that sense a 'top constant'; $\perp$ is a local 'bottom constant': ${ }^{4}$ it entails every sentence of $\mathcal{L}_{S}$, but not every sentence of some extensions of $\mathcal{L}_{S} \cdot{ }^{15}$ Similarly, $\sim$ functions locally as classical negation. It is easily seen that, for every domain $\mathrm{D}$, there is a trivial model $M=\langle\mathrm{D}, v\rangle$, a model such that $v_{M}(A)=I$ for all $A \in \mathcal{W}_{S}$. The following tables obtain in non-trivial models - all $\{\neg, \sim\}$-combinations are equivalent to one of these:

\begin{tabular}{c||c|c|c|c|c|c} 
& $\neg$ & $\sim$ & $\sim \sim$ & $\neg \sim$ & $\sim \neg$ & $\sim \sim \neg$ \\
\hline$T$ & $F$ & $F$ & $T$ & $T$ & $T$ & $F$ \\
$I$ & $I$ & $F$ & $T$ & $T$ & $F$ & $T$ \\
$F$ & $T$ & $T$ & $F$ & $F$ & $F$ & $T$
\end{tabular}

$$
\begin{array}{c|c}
\sim \neg A & A \text { is true and consistent } \\
A \wedge \neg A & A \text { is inconsistent } \\
\sim A & A \text { is false }
\end{array}
$$

So $M$ is inconsistent iff $v_{M}(\neg A)=v_{M}(A)$ for some $A \in \mathcal{W}_{S} ; M$ is trivial iff $v_{M}(\sim A)=v_{M}(A)$ for some $A \in \mathcal{W}_{S}$, viz. iff $v_{M}(A)=I$ for all $A \in \mathcal{W}_{S}$.

Both $A \vdash_{\mathrm{CLuNs}} \sim \neg A \vee(A \wedge \neg A)$ and $\neg A \vdash_{\mathrm{CLuNs}} \sim A \vee(A \wedge \neg A)$ hold; in words: if $A$ is true, $A$ is either consistently true or inconsistent; if $\neg A$ is true, $A$ is either false or inconsistent. Also $A \supset \top, \sim \perp$, and $\sim \neg A \vee(A \wedge \neg A) \vee \sim A$ are CLuNs-theorems. However, $\perp \supset A$ and $\sim A \supset \neg A$ are only local CLuNstheorems; they hold in $\mathcal{L}_{S}$ but not in all extensions of $\mathcal{L}_{S}$. The same holds for $(\sim \neg A \wedge(A \wedge \neg A)) \supset B,(\sim A \wedge(A \wedge \neg A)) \supset B,(\sim \neg A \wedge \sim A) \supset B, \sim(\sim \neg A \wedge(A \wedge$ $\neg A)), \sim(\sim A \wedge(A \wedge \neg A))$ and $\sim(\sim \neg A \wedge \sim A)$. So the members of $\{\sim \neg A, A \wedge$ $\neg A, \sim A\}$ are jointly exhaustive and, locally, are mutually exclusive whenever $A \in \mathcal{W}_{S}$. As this paper concerns the AFS as set theories, local CLuNs-theorems will henceforth be called CLuNs-theorems.

Some sets will be given a special name. ${ }^{16}$

$$
\begin{array}{rlll}
\emptyset==_{d f} & \{x \mid \perp\} \\
U==_{d f} & \{x \mid \top\} \\
G==_{d f} & \{x \mid \exists x \exists y(y \in x \wedge y \notin x)\} \\
R==_{d f} & \{x \mid x \notin x\} \\
Z={ }_{d f} & \{x \mid x \in x\} \\
Q_{n}=d_{d f} & \left\{x \mid \neg \exists y_{1} \ldots \exists y_{n}\left(x \in y_{1} \wedge y_{1} \in y_{2} \wedge \ldots \wedge y_{n} \in x\right)\right\} \quad(1 \leq n) \\
D=d_{d f} & \{x \mid x \neq x\} \\
\emptyset^{\circ}={ }_{d f} & \{x \mid \forall y(x=y \wedge x \neq y \wedge x \in y \wedge x \notin y)\} \\
\emptyset^{\Delta}={ }_{d f} & \{x \mid \forall y x \in y\} \\
U^{\Delta}={ }_{d f} & \{x \mid \exists y x \in y\} \\
D^{*}={ }_{d f} & \{x \mid \sim x=x\} \\
R^{*}={ }_{d f} & \{x \mid \sim x \in x\} \\
Q_{n}^{*}=d_{d f} & \left\{x \mid \sim \exists y_{1} \ldots \exists y_{n}\left(x \in y_{1} \wedge y_{1} \in y_{2} \wedge \ldots \wedge y_{n} \in x\right)\right\} \quad(1 \leq n) \\
N_{0}==_{d f} & \{x \mid \sim \exists y y \in x\} \\
N_{1}==_{d f} & \{x \mid \exists y(y \in x \wedge \forall z(z \in x \supset z=y))\}
\end{array}
$$

\footnotetext{
${ }^{14}$ It was shown $[5$, p. $25 \mathrm{sub}(\mathrm{C})]$ that a local bottom constant is also definable within several interesting paraconsistent arithmetic theories.

${ }^{15}$ This refers to the logic. In the context of PFS and AFS the situation is more complicated and studied elsewhere.

${ }^{16}$ Sets only exist by virtue of the axiom schema Abs - see the next section; the definition sign merely indicates renaming or abbreviation.
} 


$$
\begin{gathered}
N_{n}={ }_{d f} \quad\left\{x \mid \exists y_{1} \ldots \exists y_{n}\left(\sim y_{1}=y_{2} \wedge \ldots \wedge \sim y_{1}=y_{n} \wedge \ldots \wedge \sim y_{n-1}=y_{n} \wedge\right.\right. \\
y_{1} \in x \wedge \ldots \wedge y_{n} \in x \wedge \\
\left.\left.\forall z\left(z \in x \supset\left(z=y_{1} \vee \ldots \vee z=y_{n}\right)\right)\right)\right\} \quad(n \geq 2) \\
C={ }_{d f} \quad\{x \mid \forall y \sim(y \in x \wedge y \notin x)\}
\end{gathered}
$$

Set $G$ will turn out remarkable; $D, \emptyset^{\circ}$ and $\emptyset^{\Delta}$ are variants of the empty set $\emptyset$; $U^{\Delta}$ is a variant of the universal set $U$. The presence of $\sim$ warrants the existence of the variant $D^{*}$ and of the 'classical' Russell set $R^{*}$. Remarkably, $R^{*}$ does not cause triviality — see Theorem 6 in Section 5. As $\vdash_{\text {CLuNs }} \forall x \forall y \neg(y \in x \wedge y \notin x)$, the touchstone of $C$, the set of consistent sets, requires classical negation.

If a CLuNs-theory in the language $\mathcal{L}_{S}$ is inconsistent, some of its theorems are disjunctions of formulas of the form $\mathrm{Q}\left(t_{1}=t_{2} \wedge t_{1} \neq t_{2}\right)$ or $\mathrm{Q}\left(t_{1} \in t_{2} \wedge t_{1} \notin t_{2}\right)$. So the theory requires the existence of sets that are different from themselvesbecause $\alpha=\beta, \alpha \neq \beta \vdash_{\text {CLuNs }} \alpha \neq \alpha$-or the existence of sets of which some entities are both members and non-members. The CLuNs-theory PFS, defined in the next Section, has no theorems of the form $\mathrm{Q} \alpha \neq \beta$; see Theorem 4 , but PFS has inconsistent sets - $t$ is an inconsistent set iff $\exists x(x \in t \wedge x \notin t)$ holds.

Consider some definable operators, first some subset relations.

$$
\begin{aligned}
& t_{1} \subseteq t_{2} \quad=_{d f} \quad \forall z\left(z \in t_{1} \rightarrow z \in t_{2}\right) \\
& t_{1} \varsubsetneqq t_{2} \quad=_{d f} \quad \forall z\left(\left(z \in t_{1} \rightarrow z \in t_{2}\right) \wedge t_{1} \neq t_{2}\right) \\
& t_{1} \subset t_{2} \quad{ }_{d f} \quad \forall z\left(\left(z \in t_{1} \rightarrow z \in t_{2}\right) \wedge \sim t_{1}=t_{2}\right)
\end{aligned}
$$

Replacing $\rightarrow$ by $\supset$, one obtains weaker relations which have rather uninteresting properties. If an AFS has no models that verify $\exists x x \neq x$, then it has $\forall\left(t_{1} \varsubsetneqq t_{2} \leftrightarrow\right.$ $\left.t_{1} \subset t_{2}\right)$ as a theorem.

$$
\begin{aligned}
& \bar{t} \quad{ }_{d f} \quad\{x \mid x \notin t\} \\
& \tilde{t}=d f \quad\{x \mid \sim x \in t\} \\
& t_{1}-t_{2} \quad{ }_{d f} \quad\left\{x \mid x \in t_{1} \wedge x \notin t_{2}\right\} \\
& t_{1} \backslash t_{2} \quad{ }_{d f} \quad\left\{x \mid x \in t_{1} \wedge \sim x \in t_{2}\right\} \\
& \left\{t_{1}, \ldots, t_{n}\right\}={ }_{d f} \quad\left\{x \mid x=t_{1} \vee \ldots \vee x=t_{n}\right\} \quad(n \geq 1) \\
& \bigcap(t)={ }_{d f} \quad\{x \mid \forall y(y \in t \supset x \in y)\} \\
& \bigcup(t)={ }_{d f} \quad\{x \mid \exists y(y \in t \wedge x \in y)\}^{17} \\
& \wp(t)={ }_{d f} \quad\{x \mid x \subseteq t\} \\
& i(t) \quad{ }_{d f} \quad\{x \mid x \in t\} \\
& \oplus(t)=d_{d f} \quad\{x \mid \sim \sim x \in t\} \\
& \ominus(t)={ }_{d f} \quad\{x \mid \sim \sim x \notin t\} \\
& \boxplus\{x \mid A(x)\} \quad{ }_{d f} \quad\{x \mid \sim \sim(x \in\{x \mid A(x)\} \wedge A(x))\} \\
& \boxminus\{x \mid A(x)\} \quad{ }_{d f} \quad\{x \mid \sim \sim(x \notin\{x \mid A(x)\} \wedge \neg A(x))\}
\end{aligned}
$$

These operations seem self-explanatory but their precise meaning obviously depends on the Abstraction axiom, by virtue of which sets exist. ${ }^{18}$

\footnotetext{
${ }^{17}$ Where $t=\left\{t_{1}, \ldots, t_{n}\right\}$, I shall also write $t_{1} \cap \ldots \cap t_{n}$ and $t_{1} \cup \ldots \cup t_{n}$.

${ }^{18}$ Ordered pairs and sequences are defined as usual in terms of sets, but will not be given any special attention in this paper.
} 


\section{The Axioms and Elementary Considerations}

Desideratum 1 forces us to make some choices. To the detachable and contraposable material implication of CL correspond three different implications in CLuNs, all introduced before: $A \sqsupset B, A \supset B$ and $A \rightarrow B$; their meanings as specified within CLuNs coincide in CL. Similarly, the material equivalence of $\mathbf{C L}$ corresponds to a manifold of different equivalences in CLuNs. A perspicuous view on the matter sees an equivalence as the conjunction of four implications: $A \rightsquigarrow_{1} B, \neg A \rightsquigarrow_{2} \neg B, B \rightsquigarrow_{3} A$ and $\neg B \rightsquigarrow_{4} \neg A$, each of the four squiggly arrows being either the non-detachable $\sqsupset$ or the detachable $\supset$. So Frege's axioms may provisionally be rendered as

$$
\begin{gathered}
\forall x \forall y\left(\forall z\left(z \in x \rightsquigarrow_{1} z \in y\right) \rightsquigarrow_{5} x=y\right) \\
\forall \beta\left(\beta \in\{\alpha \mid A(\alpha)\} \rightsquigarrow_{2} A(\beta)\right)
\end{gathered}
$$

with (2) the conjunction of $(3)-(6)$ :

$$
\begin{aligned}
& \forall \beta\left(\beta \in\{\alpha \mid A(\alpha)\} \rightsquigarrow_{1} A(\beta)\right) \\
& \forall \beta\left(\beta \notin\{\alpha \mid A(\alpha)\} \rightsquigarrow_{2} \neg A(\beta)\right) \\
& \forall \beta\left(A(\beta) \rightsquigarrow_{3} \beta \in\{\alpha \mid A(\alpha)\}\right) \\
& \forall \beta\left(\neg A(\beta) \rightsquigarrow_{4} \beta \notin\{\alpha \mid A(\alpha)\}\right)
\end{aligned}
$$

The following heuristic journey leads to the choices.

(A) There are inconsistent sets in PFS. Consider the $R$-instance of (2) for $R$ : $R \in R \rightsquigarrow_{2} R \notin R$. For both choices of $\rightsquigarrow_{1}$ and $\rightsquigarrow_{2}$, the corresponding instance of (3) entails $R \notin R$ and the corresponding instance of (4) entails $R \in R$.

(B) The 'collection' of non-members of an inconsistent set is not a function of the 'collection' of members. So, in the presence of inconsistent sets, one needs both collections in order to characterize a set.

(C) By a reasoning as in (A), both $R^{*} \in R^{*}$ and $R^{*} \notin R^{*}$ are PFS-theorems. So $\rightsquigarrow_{1}$ cannot be $\supset$; if it were, the relevant instance of $(3)$ would entail $\sim R^{*} \in$ $R^{*}$, causing PFS to be trivial.

(D) Let $X$ abbreviate $\{x \mid \sim \sim x \notin x\}$. By a reasoning as in (A), both $X \notin X$ and $X \in X$ are PFS-theorems. So $\rightsquigarrow_{2}$ cannot be $\supset$; if it were, the relevant instance of (4) would entail $\sim X \notin X$, causing PFS to be trivial.

(E) That $\rightsquigarrow_{3}$ and $\rightsquigarrow_{4}$ are detachable does not cause triviality - see Theorem 6. So they should be detachable. Indeed, it is Frege's basic idea that $A(t)$ is sufficient for $t \in\{x \mid A(x)\}$ and that $\neg A(t)$ is sufficient for $t \notin\{x \mid A(x)\}$-but see Section 9 on $\mathrm{Abs}^{\Leftrightarrow}$.

(F) In view of Desideratum 2, $\rightsquigarrow 1$ should be the contraposable equivalence $\leftrightarrow$ rather than $\equiv^{19}$ and $\rightsquigarrow_{5}$ should be detachable.

So we have settled for the following axiom and schema:

Ext $\forall x \forall y(\forall z(z \in x \leftrightarrow z \in y) \supset x=y)$

Abs $\forall \beta(\beta \in\{\alpha \mid A(\alpha)\} \Longleftrightarrow A(\beta))$

\footnotetext{
${ }^{19}$ Any other choice is ridiculous; e.g., we want neither $\vdash_{\mathbf{P F S}} G=U$ nor $\vdash_{\text {AFS }}\{x \mid A(x)\}=$ $\{x \mid A(x) \wedge \exists y(y \in y \wedge y \notin y)\}$.
} 
PFS-models $M=\langle\mathrm{D}, v\rangle$ have the following properties. In view of Ext: if $o_{1}$ and $o_{2}$ are distinct elements of $\mathrm{D}$, then there is an $o_{3} \in \mathrm{D}$ such that, for at least two $X \in\{T, I, F\}$, if $\left\langle o_{3}, o_{1}\right\rangle \in v^{X}(\in)$ then $\left\langle o_{3}, o_{2}\right\rangle \notin v^{X}(\in)$. In view of Abs: for all $\alpha \in \mathcal{O}$, (i) if $M \Vdash A(\alpha)$ then $\left\langle v(\alpha), v(\{\beta \mid A(\beta)\}\rangle \in v^{T}(\in) \cup v^{I}(\in)\right.$ and (ii) if $M \Vdash \neg A(\alpha)$ then $\left\langle v(\alpha), v(\{\beta \mid A(\beta)\}\rangle \in v^{I}(\in) \cup v^{F}(\in)\right.$.

Define $\{\alpha \mid A(\alpha)\}$ as clean in PFS-model $M$ iff $M \Vdash \forall \beta(\beta \in\{\alpha \mid A(\alpha)\} \leftrightarrow$ $A(\beta)) ;\{\alpha \mid A(\alpha)\}$ is unclean with respect to membership in $M$ iff $M \Vdash \exists \beta(\beta \in$ $\{\alpha \mid A(\alpha)\} \wedge \sim A(\beta))$; it is unclean with respect to non-membership in $M$ iff $M \Vdash \exists \beta(\beta \notin\{\alpha \mid A(\alpha)\} \wedge \sim \neg A(\beta))$. The set $\{\alpha \mid A(\alpha)\}$ is unclean with respect to membership in set theory $\mathbf{S}$ iff $\vdash_{\mathbf{S}} \exists \beta(\beta \in\{\alpha \mid A(\alpha)\} \wedge \sim A(\beta))$; similarly for non-membership iff $\vdash_{\mathbf{S}} \exists \beta(\beta \notin\{\alpha \mid A(\alpha)\} \wedge \sim \neg A(\beta))$. In view of $(\mathrm{C}), R^{*}$ is unclean with respect to membership in PFS: $\vdash_{\text {PFS }} R^{*} \in R^{*} \wedge \sim \sim R^{*} \in R^{*} .^{20}$ In (D), $\{x \mid \sim \sim x \notin x\}$ is shown unclean with respect to non-membership in PFS. With the exception of abstracting terms that define sets identical to $G$, all abstracting terms are unclean in some non-trivial PFS-models. By going adaptive one may eliminate PFS-models in which terms are needlessly unclean, for example models verifying $\emptyset \in \emptyset$, or in which unclean terms are more unclean than required, for example models verifying $R \in R^{*}$. One may call $t_{1}$ a clean or unclean (non-)member of $t_{2}$; thus $R$ is an unclean member of $\emptyset$ if $R \in \emptyset$ and is an unclean non-member of $U$ if $R \notin U$.

Let us have a closer look at PFS, keeping in mind that the ultimate aim is an adequate AFS.

In view of RoI, replacing the implication in Ext by the standard equivalence $\equiv$ results in a PFS-theorem, but replacing it by the contraposable $\leftrightarrow$ does not. The latter replacement would cause inconsistent sets to be different from all sets, including themselves. The feature, which holds in Zach Weber's dialetheic set theory [26, 27, 28], comes to an idle spreading of inconsistency in PFS. ${ }^{21}$

As abstracting terms are unclean in some PFS-models, several elements of the domain may function as the assignment value of the same abstracting term. So, where $t \in \mathcal{T}^{c}$, some models differ only in $v(t)$. Going adaptive, one may eliminate PFS-models in which terms are more unclean than required.

The PFS-theorems $\forall x x \notin \emptyset$ and $\forall x x \in U$ are CLuNs-equivalent to $\neg \exists x x \in$ $\emptyset$ and $\neg \exists x x \notin U$ respectively. We obviously want $\sim \exists x x \in \emptyset$ and $\sim \exists x x \notin U$ as AFS-theorems; only these warrant that $\emptyset$ and $U$ are clean.

The maximally inconsistent set $G$ is remarkable. The abstracting terms that are PFS-provably identical to $G,{ }^{22}$ are the only terms that are clean in all nontrivial models as well as, contingent on $\mathrm{D}$, invariant over all models. As an effect, $\vdash_{\text {PFS }} \forall x(\forall y(y \in x \wedge y \notin x) \equiv x=G)$.

As $R \in R$ and $R \notin R$ are PFS-theorems, so are $R \in Z$ and $R \notin Z$. None of the following four are PFS-theorems: $Z \in Z, Z \notin R, Z \notin Z$, and $Z \in R$. Moreover, we do not want any of them as AFS-theorems because there apparently are no reasons to prefer either the former two or the latter two. So

\footnotetext{
${ }^{20}$ On the one hand, $R^{*}$ is defined within PFS in the same way as $R$ is defined in Frege's original set theory and the negation in $R^{*}$ is classical within $\mathcal{L}_{S}$. On the other hand, $R^{*}$ has some members for which the touchstone does not hold; if it did, PFS would be trivial. So whether, within PFS, the Russell set $R^{*}$ is called classical is partly a matter of convention.

${ }^{21}$ Yet, the complicated identity is definable in PFS, for example, by $t_{1} \bumpeq t_{2}={ }_{d f} \forall x(x \in$ $\left.t_{1} \leftrightarrow x \in t_{2}\right)$. This gives $\vdash_{\mathbf{P F S}} t_{1} \bumpeq t_{2} \equiv t_{1}=t_{2}$ and $\vdash_{\mathbf{P F S}} \neg t_{1} \bumpeq t_{2} \equiv\left(t_{1} \neq t_{2} \vee \exists x(x \in\right.$ $\left.t_{1} \wedge x \notin t_{1}\right)$ )- sic. In view of Theorem 4 below in the text, it is easy enough to ensure that an AFS has all formulas $\sim Q\left(t_{1}=t_{2} \wedge t_{1} \neq t_{2}\right)$ as theorems-see $\mathbf{A F S} \mathbf{S}_{2}$ in Section 8 .

${ }^{22}$ If $\nvdash_{\text {PFS }} t=G$, then $t=G$ is not a theorem of any AFS.
} 
$Z$ is a set that we want to be underdetermined by the theory, a set that should be a member of itself in some models and a non-member of itself in others.

The sets $Q_{n}(n \geq 1)$ are Quine's [18] variants to the Russell set. The following CLuNs-proof for $Q_{1}$ is easily generalized to all $Q_{n}$.

$\begin{array}{lll}1 & \forall x\left(x \in Q_{1} \Longleftrightarrow \neg \exists y(x \in y \wedge y \in x)\right. & \text { Abs } \\ 2 & Q_{1} \in Q_{1} \Longleftrightarrow \neg \exists y\left(Q_{1} \in y \wedge y \in Q_{1}\right) & 1 \\ 3 & Q_{1} \in Q_{1} \Longleftrightarrow \neg\left(Q_{1} \in Q_{1} \wedge Q_{1} \in Q_{1}\right) & 2 \\ 4 & Q_{1} \in Q_{1} \Longleftrightarrow Q_{1} \notin Q_{1} & 3 \\ 5 & Q_{1} \notin Q_{1} \supset Q_{1} \in Q_{1} & 4 \\ 6 & Q_{1} \notin Q_{1} \vee Q_{1} \notin Q_{1} & 4 \\ 7 & Q_{1} \in Q_{1} & 5 \\ 8 & Q_{1} \notin Q_{1} & 6\end{array}$

We obviously want $D, D^{*}, \emptyset^{\circ}, \emptyset^{\Delta}$ and $\emptyset$ to be identical in AFS, but they are not provably identical in PFS. Indeed, there are models in which some of the terms are unclean and others clean, or more unclean or less unclean.

Each of the following are PFS-theorems: $R^{*} \in R, R^{*} \notin R$; for all $i \geq 1$, $Q_{i}^{*} \in Q_{i}^{*}, Q_{i}^{*} \notin Q_{i}^{*}, Q_{i} \in R, Q_{i} \notin R, Q_{i}^{*} \in R$ and $Q_{i}^{*} \notin R ; R \notin R^{*}$ and, for all $i \geq 1, Q_{i} \notin R^{*}$ and $Q_{i}^{*} \notin R^{*}$. The following non-PFS-theorems are desirable as AFS-theorems: $\sim R \in R^{*}$ and, for all $i \geq 1, \sim Q_{i} \in R^{*}$ and $\sim Q_{i}^{*} \in R^{*}$.

The $N_{i}$ are the sets of sets with $i$ members: $N_{0}$ the set of empty sets, $N_{1}$ the set of singletons, etc. The occurrence of the classical negation is essential: $\vdash_{\mathbf{P F S}} G \in\{x \mid \neg \exists y y \in x\}$ but we want $\sim G \in N_{0}$ in all AFS.

While $\bar{t}$ depends on the members of $t$ as well as on the non-members of $t, \widetilde{t}$ depends only on the members of $t$. A similar comment applies to $t_{1}-t_{2}$ and $t_{1} \backslash t_{2}$. An extreme case is $i(t)$ : whether $t$ is clean or not, every member (non-member) of $t$ is a member (non-member) of $i(t)$, but $i(t)$ may have further members as well as further non-members in PFS-models in which it is unclean. Sets $\oplus(t), \ominus(t), \boxplus\{x \mid A(x)\}$ and $\boxminus\{x \mid A(x)\}$ are consistent iff clean-note that $\nvdash_{\mathbf{P F S}} R \notin \oplus(R)$ and $\nvdash_{\mathbf{P F S}} R^{*} \in \boxplus\left(R^{*}\right)$.

Sets are extremely 'malleable'. Expressions such as $\{x \mid A(x) \vee(x=t \wedge$ $\exists x !(x \in x))\},\{x \mid x \in\{x \mid A(x)\} \vee(x=t \wedge \exists x !(x \in x))\}$ and $\{x \mid A(x)\} \cup(\{t\} \cap G)$ enable one to define a set exactly like $\{x \mid A(x)\}$ but with $t$ a member of it. Other expressions enable one to define a set exactly like $\{x \mid A(x)\}$ but with $t$ a non-member of it. Where $t_{1} \in t_{2} \wedge t_{1} \notin t_{2}$, one may define a $t_{3}$ which, in AFS, is exactly like $t_{2}$ except that $\sim t_{1} \in t_{3}$ or that $\sim t_{1} \notin t_{3}$ as one prefers. Instead of defining a set by 'adding' or 'removing' $t_{1}$ as a member or non-member, one may, by similar means, add or remove the members of $t_{1}$ as members or non-members.

\section{Some Models}

Consider first $M_{1}^{1}$, a trivial PFS-model. Its singleton domain and assignment function may, with notational abuse, be summarized as follows.

$$
\begin{aligned}
& M_{1}^{1} \quad \mathrm{D}=\{m\} \\
& \begin{array}{ll|l}
\text { for all } t \in \mathcal{T}^{c} \cup \mathcal{O}, v(t)=m \quad \frac{m}{m} & =, \neq
\end{array} \quad \begin{array}{l|l}
m \\
& \in, \notin
\end{array}
\end{aligned}
$$


Spelling out the predicates: $v^{T}(\in)=v^{F}(\in)=v^{T}(=)=v^{F}(=)=\emptyset$ and $v^{I}(\in)=$ $v^{I}(=)=\{\langle m, m\rangle\}$. As all abstracting terms are mapped to $m, G$ is clean and terms $t$ for which $\nvdash_{\mathbf{P F S}} t=G$ are maximally unclean in $M_{1}^{1}$. The model verifies all sentences of $\mathcal{L}_{S}$. Moreover, it is a collapsed model of Fregean set theory. The term is taken from collapsed models of arithmetic $[5,14,15,16,21,22]$. However, arithmetic is presumed to be consistently given by the Peano axioms, whereas the adaptive Fregean set theory is inconsistent as well as under construction.

Let model $M_{2}^{1}$ be exactly like $M_{1}^{1}$ except in that $v^{T}(=)=\{\langle m, m\rangle\}$ whereas $v^{I}(=)=v^{F}(=)=\emptyset$. Clearly $M_{2}^{1}$ verifies $\forall x \forall y x=y, \forall x \forall y x \in y$ and $\forall x \forall y x \notin$ $y$. So $M_{2}^{1}$ verifies Ext as well as all instances of Abs. ${ }^{23}$

Theorem 4 No PFS-theorem has the form $\mathbf{Q} \alpha \neq \beta$.

Proof. $M_{2}^{1}$ is a PFS-model and $M_{2}^{1} \nVdash \exists x \exists y x \neq y$.

Theorem 5 No PFS-theorem has the form $\mathbf{Q} \sim \alpha \in \beta$.

Proof. $M_{2}^{1}$ is a PFS-model, $M_{2}^{1} \nVdash \perp$ and $M_{2}^{1} \Vdash \forall x \forall y x \in y$.

Theorem 6 PFS is non-trivial.

Proof. $M_{2}^{1}$ is a PFS-model and $M_{2}^{1} \nVdash \perp$.

Proofs of Strong Reassurance occur in several places [3, Theorem 4]. Applied to AFS, this comes to: if PFS is non-trivial, no AFS is trivial. ${ }^{24}$

Theorem 7 All AFS are non-trivial.

Let the domain and assignment of PFS-model $M_{1}^{3}$ be as follows.

$M_{1}^{3}$

$$
\begin{aligned}
& v(\emptyset)=l \\
& v(U)=u \\
& v(t)=m \text { otherwise }^{25}
\end{aligned}
$$

$$
\mathrm{D}=\{l, m, u\}
$$

\begin{tabular}{c|ccccc|ccc} 
& $l$ & $m$ & $u$ & & $l$ & $m$ & $u$ \\
\hline$l$ & $=$ & $\neq$ & $\neq$ & $l$ & $\notin$ & $\in, \notin$ & $\epsilon$ \\
$m$ & $\neq$ & $=, \neq$ & $\neq$ & & $m$ & $\notin$ & $\in, \notin$ & $\epsilon$ \\
$u$ & $\neq$ & $\neq$ & $=$ & & $\notin$ & $\notin$ & $\epsilon, \notin$ & $\epsilon$
\end{tabular}

The tables summarize: $v^{T}(\in)=\{\langle l, u\rangle,\langle m, u\rangle,\langle u, u\rangle\}, v^{I}(\in)=\{\langle l, m\rangle,\langle m, m\rangle$, $\langle u, m\rangle\}, v^{F}(\in)=\{\langle l, l\rangle,\langle m, l\rangle,\langle u, l\rangle\}, v^{T}(=)=\{\langle l, l\rangle,\langle u, u\rangle\}, v^{I}(=)=\{\langle m, m\rangle\}$, and $v^{F}(=)$ comprises the six other couples.

To see that Ext is verified, note that $l, m$, and $u$ are different from each other with respect to the combination of their 'positive extension' (members) and 'negative extension' (non-members):

\begin{tabular}{c|c|c} 
& $\in$ & $\notin$ \\
\hline$l$ & & $l, m, u$ \\
$m$ & $l, m, u$ & $l, m, u$ \\
$u$ & $l, m, u$ &
\end{tabular}

\footnotetext{
${ }^{23}$ These are, for every $A(x): \forall y(A(y) \supset y \in\{x \mid A(x)\}), \forall y(\neg A(y) \supset y \notin\{x \mid A(x)\})$, $\forall y(y \notin\{x \mid A(x)\} \vee A(y))$ and $\forall y(y \in\{x \mid A(x)\} \vee \neg A(y))$.

${ }^{24}$ In semantic terms, Strong Reassurance (also called Stopperedness or Smoothness) comes to this: for all adaptive logics in standard format, if a premise set has a lower limit model $M$ that is not minimally abnormal, then the premise set has a minimally abnormal lower limit model $M^{\prime}$ such that $A b\left(M^{\prime}\right) \subset A b(M)$. So as PFS has non-trivial models (by Theorem 6), every AFS has non-trivial models. The essential step in the proof is the absence of infinitely descending chains of abnormal models.

25 "Otherwise" abbreviates "for all $t \in \mathcal{O} \cup \mathcal{T}^{c}-\{\emptyset, U\}$; similarly for other models below in the text. Equivalent touchstones do not warrant identical abstracting terms.
} 
Abs is also verified. The following table lists, in the first two rows, the couples $\left\langle S_{1}, S_{2}\right\rangle$ for which $S_{1} \cup S_{2}=\mathrm{D}$. The third row mentions the elements of $\mathrm{D}$ that have all of $S_{1}$ as members and all of $S_{2}$ as non-members. Please check that the assignment to abstracting terms agrees with this.

\begin{tabular}{c||c|c|c|c|c|c|c|c|c|c||}
\hline$\in t$ & - & $l$ & $l$ & $m$ & $\ldots$ & $m, u$ & $l, m, u$ & $\ldots$ & $l, m, u$ & $l, m, u$ \\
$\notin t$ & $l, m, u$ & $m, u$ & $l, m, u$ & $l, u$ & $\ldots$ & $l$ & $l, m, u$ & $\ldots$ & $u$ & - \\
\hline$v(t)$ & $l / m$ & $m$ & $m$ & $m$ & $\ldots$ & $m$ & $m$ & $\ldots$ & $m$ & $m / u$ \\
\hline
\end{tabular}

In $M_{1}^{3}$, the sets $\emptyset$ and $U$ are clean and so are all $t$ such that $\vdash_{\mathbf{P F S}} t_{1}=G$; all other abstracting terms are unclean.

Let $M_{2}^{3}$ be exactly like $M_{1}^{3}$ except that identity is consistent and $\langle m, m\rangle \in$ $v^{T}(=)$. This model is just as much a PFS-model as $M_{1}^{3}$ and showing that it is proceeds as for $M_{1}^{3}$.

I mention some more PFS-models; some will be referred to in the sequel.

$M_{1}^{2}$

$$
\begin{aligned}
& v(\emptyset)=l \\
& v(t)=m \text { otherwise }
\end{aligned}
$$

$$
\mathrm{D}=\{l, m\}
$$

\begin{tabular}{c|cc} 
& $l$ & $m$ \\
\hline$l$ & $\notin$ & $\in, \notin$ \\
$m$ & $\notin$ & $\in, \notin$
\end{tabular}

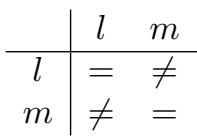

$M_{2}^{2}$

$$
\mathrm{D}=\{m, u\}
$$

$$
\begin{aligned}
& v(U)=u \\
& v(t)=m \text { otherwise }
\end{aligned}
$$

\begin{tabular}{l|ll} 
& $m$ & $u$ \\
\hline$m$ & $=$ & $\neq$ \\
$u$ & $\neq=$
\end{tabular}

$M_{1}^{4}$

$$
\mathrm{D}=\{l, m, r, u\}
$$

$$
\begin{aligned}
& v(R)=r \\
& v(U)=u \\
& v(t)=m \text { otherwise }
\end{aligned}
$$

\begin{tabular}{c|cccc} 
& $l$ & $m$ & $r$ & $u$ \\
\hline$l$ & $\notin$ & $\in, \notin$ & $\in$ & $\in$ \\
$m$ & $\notin$ & $\in, \notin$ & $\in, \notin$ & $\in$ \\
$r$ & $\notin$ & $\in, \notin$ & $\in, \notin$ & $\in$ \\
$u$ & $\notin$ & $\in, \notin$ & $\notin$ & $\in$
\end{tabular}

\begin{tabular}{c|cccc} 
& $l$ & $m$ & $r$ & $u$ \\
\hline$l$ & $=$ & $\neq$ & $\neq$ & $\neq$ \\
$m$ & $\neq$ & $=$ & $\neq$ & $\neq$ \\
$r$ & $\neq$ & $\neq$ & $=$ & $\neq$ \\
$u$ & $\neq$ & $\neq$ & $\neq$ & $=$
\end{tabular}

$M_{2}^{4}$

$$
\mathrm{D}=\{l, m, r, u\}
$$

$$
\begin{aligned}
& v(R)=r \\
& v(U)=u \\
& v(t)=m \text { otherwise }
\end{aligned}
$$

\begin{tabular}{c|cccc} 
& $l$ & $m$ & $r$ & $u$ \\
\hline$l$ & $\notin$ & $\in, \notin$ & $\in, \notin$ & $\in$ \\
$m$ & $\notin$ & $\in, \notin$ & $\in, \notin$ & $\in$ \\
$r$ & $\notin$ & $\in, \notin$ & $\in, \notin$ & $\in$ \\
$u$ & $\notin$ & $\in, \notin$ & $\notin$ & $\in$
\end{tabular}

\begin{tabular}{c|cccc} 
& $l$ & $m$ & $r$ & $u$ \\
\hline$l$ & $=$ & $\neq$ & $\neq$ & $\neq$ \\
$m$ & $\neq$ & $=$ & $\neq$ & $\neq$ \\
$r$ & $\neq$ & $\neq$ & $=$ & $\neq$ \\
$u$ & $\neq$ & $\neq$ & $\neq$ & $=$
\end{tabular}

Checking that $M_{1}^{4}$ and $M_{2}^{4}$ are PFS-models proceeds as for $M_{1}^{3}$ and is just as easy. Model $M_{2}^{4}$ verifies $\exists x(x \notin R \wedge \sim x \in x)$ whereas $M_{1}^{4}$ falsifies it - actually $R$ is clean in $M_{1}^{4}$. As no abstracting term names $l$ in either model, there is no abstracting term $t$ such that $M_{2}^{4} \Vdash t \notin R \wedge \sim t \in t$ and $M_{1}^{4} \nVdash t \notin R \wedge \sim t \in t$.

\section{Abnormalities Revisited}

A set that is unclean in a model is inconsistent in that model. Yet, a set may be inconsistent as well as clean in a model: $M_{1}^{4} \Vdash \exists x(x \in R \wedge x \notin R)$ 
but $M_{1}^{4} \Vdash \forall x(x \in R \leftrightarrow x \notin x)$. In $M_{2}^{4}$, to the contrary, $R$ is inconsistent and unclean, $M_{2}^{4} \Vdash \exists x(x \notin R \wedge \sim x \in x)$. This seems a good reason to prefer $M_{1}^{4}$ over $M_{2}^{4},{ }^{26}$ but $\mathbf{C L u N s}{ }^{m}$ does not make such preference: $A b\left(M_{2}^{4}\right)=A b\left(M_{1}^{4}\right)$ - the models verify the same non-quantified formulas because no constant term names $l$; they verify the same quantified formulas because the effect of $\langle l, r\rangle \in v^{I}(\in)$ is that $M_{2}^{4} \Vdash \exists x(x \in R \wedge x \notin R)$, but $M_{1}^{4}$ and $M_{1}^{4}$ verify the same formula in view of $\langle r, r\rangle \in v^{I}(\in)$. In order that $M_{1}^{4}$ be preferred over $M_{2}^{4}$, formulas of the forms (7) and (8) should count as abnormalities. ${ }^{27}$ Call these new abnormalities as opposed to the old ones. Some examples: $\emptyset \in Z \wedge \sim \emptyset \in \emptyset, \exists x(x \in \emptyset \wedge \sim \perp)$, $\emptyset \notin R \wedge \sim \neg \emptyset \notin \emptyset$ and $\emptyset \notin\{\emptyset\} \wedge \sim \emptyset \neq \emptyset$.

$$
\begin{array}{r}
\mathrm{Q}(t \in\{y \mid A(y)\} \wedge \sim A(t)) \\
\mathrm{Q}(t \notin\{y \mid A(y)\} \wedge \sim \neg A(t))
\end{array}
$$

Model $M_{1}^{4}$ is a far cry of a minimal abnormal $\mathbf{C L u N s}{ }^{m}$-model of PFS, so let me strengthen the argument. By present insights $R$ is clean in some minimal abnormal CLuNs ${ }^{m}$-models of PFS; let $M_{1}$ be a non-denumerable such model, whence most members of the domain $\mathrm{D}$ are not denoted by abstracting terms. Let $M_{2}$ be exactly like $M_{1}$ except in that a member of $\mathrm{D}$ that is not named by an abstracting term is not a member of itself but is a member as well as a non-member of $R$. In terms of the old abnormalities, $A b\left(M_{2}\right)=A b\left(M_{1}\right)$, but $R$ is clean in $M_{1}$ and unclean in $M_{2}$.

A different way to present the new abnormalities is by pointing out that the transition from a non-detachable implication to a detachable one may be justified by the falsehood of the connected contradiction, as suggested in the left schema, which is (locally) equivalent to the middle one, but also by the falsehood of the specific 'abnormality', as in the right schema. ${ }^{28}$

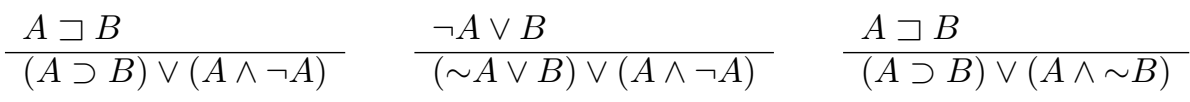

As explained in Section 4, we want to retain PFS-models verifying $Z \in Z$ and PFS-models verifying $Z \notin Z$ as AFS-models, but not models verifying both, which are unnecessarily inconsistent. As $M \Vdash Z \in Z \wedge Z \notin Z$ does not cause uncleanness in $M$, the old abnormalities should be retained with the new ones. So the set of abnormalities will from now on read:

$$
\begin{aligned}
\Omega=\left\{\mathrm{Q}(A \wedge \neg A) \mid A \in \mathcal{F}^{a}\right\} \cup \\
\left\{\mathrm{Q}(t \in\{y \mid A(y)\} \wedge \sim A(t)) \mid A \in \mathcal{F}_{S}\right\} \cup \\
\quad\left\{\mathrm{Q}(t \notin\{y \mid A(y)\} \wedge \sim \neg A(t)) \mid A \in \mathcal{F}_{S}\right\} .
\end{aligned}
$$

We are not yet completely home yet, but all abnormalities considered later on will belong to this $\Omega$. Let $\mathbf{C L u N s} \mathbf{s}_{\triangleleft}^{m}$ be exactly like $\mathbf{C L u N s}{ }^{m}$ except that the set of abnormalities is the $\Omega$ defined in (9).

\footnotetext{
${ }^{26}$ By present lights, Desideratum 3 rules out every uncleanness that is not provable in PFS. See Section 4 for $\vdash_{\text {PFS }} R^{*} \in R^{*} \wedge \sim \sim R^{*} \in R^{*}$.

${ }^{27}$ Strictly speaking, the form of $(7)$ is $\mathrm{Q}((t \in\{y \mid A(y)\} \sqsupset A(t)) \wedge(t \in\{y \mid A(y)\} \wedge \sim A(t))$ and similarly for (8). However, the first conjunct is an obvious consequence of Abs.

${ }^{28}$ Obviously $((A \sqsupset B) \wedge(A \wedge \sim B))$ will also do for the right disjunct of the right schema, but the complication is useless for (7) and (8).
} 


\section{The Adaptive Gain Vanishing}

Consider a stage of a CLuNs $s_{\triangleleft}^{m}$-proof from Ext and Abs. Let $Z$ abbreviate the abnormality $\exists z(z \in\{\emptyset\} \wedge \sim z=\emptyset)$. Dab-consequences and minimal $D a b$-consequences of $\{$ Ext, Abs $\}$ will be called $D a b$-theorems and minimal Dabtheorems (of PFS).

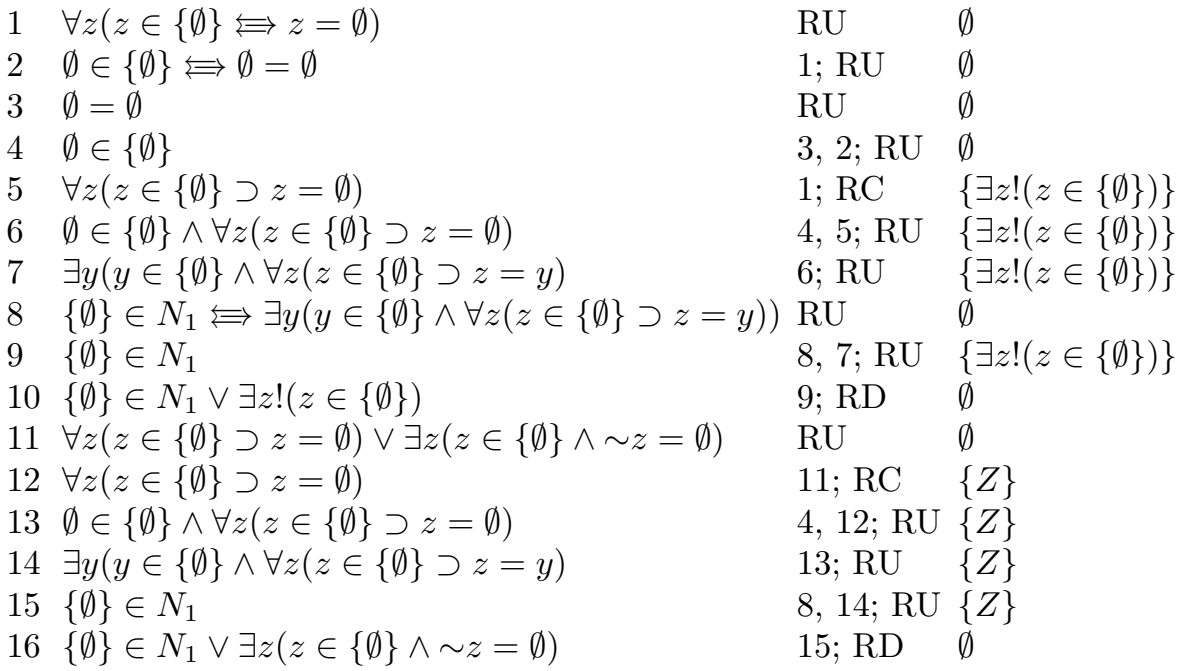

The formula of line 1 is an Abs axiom with $\{y \mid y=\emptyset\}$ replaced by $\{\emptyset\}$. The formulas of 2 and 8 are instances of Abs axioms and the formulas of 3 and 11 CLuNs-theorems. RD is a derived rule, which relies on the fact that $A$ is derivable from $\Gamma$ on condition $\Delta$ just in case $A \vee \operatorname{Dab}(\Delta)$ is derivable from $\Gamma$ on condition $\emptyset$ - see footnote 11 . Lines $11-15$ show that, in the presence of the new abnormalities, $\{\emptyset\} \in N_{1}$ is not only derivable on the condition $\{\exists z(z \in\{\emptyset\} \wedge z \notin\{\emptyset\})\}$ but also on the condition $\{\exists z(z \in\{\emptyset\} \wedge \sim z=\emptyset)\}$. According to the insights provided by the present proof stage, $\{\emptyset\} \in N_{1}$ belongs to the adaptive gain: it is conditionally derived at unmarked lines 9 and 15 .

A basic insight, due to Peter Verdée [24, 25], is that formulas only derivable on a non-empty condition from the PFS-axioms are not finally $\mathbf{C L u N s}{ }^{m}$ derivable from these axioms. The proof is related to the Curry paradox. Verdée's proof easily generalizes to $\mathbf{C L u N s} \mathbf{s}_{\triangleleft}^{m}$ and turns out to have more variants than the influenza virus. Let $C_{[A]}$ abbreviate $\{x \mid x \in x \supset A\}$ and let $K_{[A]}$ abbreviate $\{x \mid x \notin x \vee A\}$, where for the time being no variables are free in $A$.

The following four formulas are PFS-theorem schemas.

$$
\begin{gathered}
C_{[A]} \in C_{[A]} \\
A \vee !\left(C_{[A]} \in C_{[A]}\right) \\
K_{[A]} \in K_{[A]} \\
A \vee !\left(K_{[A]} \in K_{[A]}\right)
\end{gathered}
$$

I only list a proof of (12) and (13); see lines 3 and 5:

$1 \quad \forall x\left(x \in K_{[A]} \Leftrightarrow(x \notin x \vee A)\right.$

$2 \quad K_{[A]} \in K_{[A]} \Longleftrightarrow\left(K_{[A]} \notin K_{[A]} \vee A\right)$

Abs

$1 ; \mathrm{RU}$

$\emptyset$

$3 \quad K_{[A]} \in K_{[A]}$

2; RU 
$4 \quad A \vee K_{[A]} \notin K_{[A]}$

$5 \quad A \vee !\left(K_{[A]} \in K_{[A]}\right)$
$2 ; \mathrm{RU}$

3,$4 ; \mathrm{RU}$ $\emptyset$

$\emptyset$

Consider the case where a formula $A$ is derived on the condition $\Delta$, whence $A \vee \operatorname{Dab}(\Delta)$ is derivable on the empty condition - see footnote 11 . There are then several ways, all relying on (11), to derive a $D a b$-formula that has $D a b(\Delta)$ as a disjunct. I shall call them roads and number then for future reference. For the first road, I rely on the obvious fact that, if $A \notin \mathcal{F}^{a}$, then there are $A_{1}, \ldots, A_{n} \in$ $\mathcal{F}^{a}$ (for a $n \geq 1$ ) such that $A \wedge \neg A \vdash_{\mathbf{C L u N s}}\left(A_{1} \wedge \neg A_{1}\right) \vee \ldots \vee\left(A_{n} \wedge \neg A_{n}\right)$.

$\mathrm{i}+1 \quad A \vee \operatorname{Dab}(\Delta)$

$\mathrm{i}+2 \neg A \vee !\left(C_{[\neg A]} \in C_{[\neg A]}\right)$

$\mathrm{i}+3 \quad(A \wedge \neg A) \vee \operatorname{Dab}(\Delta) \vee !\left(C_{[\neg A]} \in C_{[\neg A]}\right)$

$\mathrm{i}+1, \mathrm{i}+2 ; \mathrm{RU}$

$\mathrm{i}+4 !\left(A_{1}\right) \vee \ldots \vee !\left(A_{n}\right) \vee \operatorname{Dab}(\Delta) \vee !\left(C_{[\neg A]} \in C_{[\neg A]}\right) \quad \mathrm{i}+3 ; \mathrm{RU}$

The presence of the local classical negation provides the second road:

i+1 $A \vee \operatorname{Dab}(\Delta)$

$\mathrm{i}+2 \sim A \vee !\left(C_{[\sim A]} \in C_{[\sim A]}\right)$

$\mathrm{i}+3 \quad \operatorname{Dab}(\Delta) \vee !\left(C_{[\sim A]} \in C_{[\sim A]}\right)$

And here is a third road:

$\mathrm{i}+1 \quad \operatorname{Dab}(\Delta) \vee !\left(C_{[\operatorname{Dab}(\Delta)]} \in C_{[\operatorname{Dab}(\Delta)]}\right)$ $\emptyset$

$\emptyset$

$\emptyset$

This may be applied more generally. If $B \in \Delta$ then, in view of (11),

$$
B \vee !\left(C_{[B]} \in C_{[B]}\right)
$$

is a $D a b$-theorem. Similar proofs give one the $K$-variants of these roads.

So the proof stage listed at the outset of this section may be extended, taking the formula of line 10 for $A \vee \operatorname{Dab}(\Delta)$, with a derivation of any of the following three formulas on the condition $\emptyset$.

$$
\begin{gathered}
!\left(\{\emptyset\} \in N_{1}\right) \vee \exists z !(z \in\{\emptyset\}) \vee !\left(C_{\left[\neg\{\emptyset\} \in N_{1}\right]} \in C_{\left[\neg\{\emptyset\} \in N_{1}\right]}\right) \\
\exists z !(z \in\{\emptyset\}) \vee !\left(C_{\left[\sim\{\emptyset\} \in N_{1}\right]} \in C_{\left[\sim\{\emptyset\} \in N_{1}\right]}\right) \\
\exists z !(z \in\{\emptyset\}) \vee !\left(C_{[\exists z !(z \in\{\emptyset\})]} \in C_{[\exists z !(z \in\{\emptyset\})]}\right)
\end{gathered}
$$

Similarly for the three $K$-variants.

Any such extension of the proof stage at the outset of this section causes 9 to be marked and to remain marked in all further extensions - the Dab-theorems are minimal and will remain minimal. One may proceed in the same way with respect to line 15 , identifying $A \vee \operatorname{Dab}(\Delta)$ with the formula of 16 . In this case the formulas are the following three plus their $K$-variants.

$$
\begin{gathered}
!\left(\{\emptyset\} \in N_{1}\right) \vee \exists z(z \in\{\emptyset\} \wedge \sim z=\emptyset) \vee !\left(C_{\left[\neg\{\emptyset\} \in N_{1}\right]} \in C_{\left[\neg\{\emptyset\} \in N_{1}\right]}\right) \\
\exists z(z \in\{\emptyset\} \wedge \sim z=\emptyset) \vee !\left(C_{\left[\sim\{\emptyset\} \in N_{1}\right]} \in C_{\left[\sim\{\emptyset\} \in N_{1}\right]}\right) \\
\exists z(z \in\{\emptyset\} \wedge \sim z=\emptyset) \vee !\left(C_{[\exists z(z \in\{\emptyset\} \wedge \sim z=\emptyset))]} \in C_{[\exists z(z \in\{\emptyset\} \wedge \sim z=\emptyset)]}\right)
\end{gathered}
$$

So 15 will be marked in the extensions and will remain marked if these are further extended.

An adaptive gain is also blocked by the new abnormalities. 
$\mathrm{i}+1 \quad A \vee \operatorname{Dab}(\Delta)$

$\emptyset$

$\mathrm{i}+2 C_{[\sim A]} \in C_{[\sim A]} \quad$ (10)

$\mathrm{i}+3 \quad \operatorname{Dab}(\Delta) \vee\left(C_{[\sim A]} \in C_{[\sim A]} \wedge \sim\left(C_{[\sim A]} \in C_{[\sim A]} \supset \sim A\right)\right) \quad \mathrm{i}+1, \mathrm{i}+2 ; \mathrm{RU} \quad \emptyset$

So this was the fourth road and here is the fifth road:

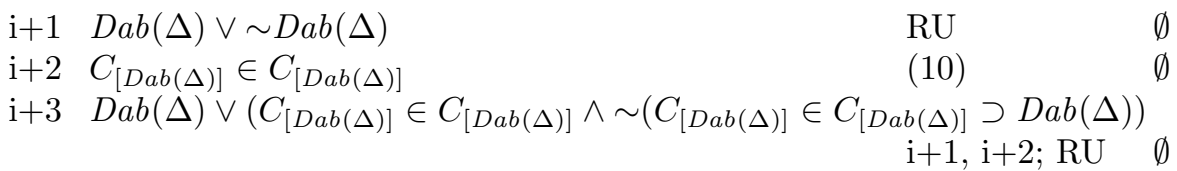

The proofs of the $K$-variants proceed similarly.

So Curry eats the adaptive gain in a most gluttonous way. There are many variants: Curry formulas with $x$ free in the touchstone, Curry formulas based on Quine sets, unions like $R \cup\{x \mid A(x)\}$, all kinds of transformations of such formulas by De Morgan-like properties and the like.

Some gluttons are unrelated to the ones discovered by Verdée. If, for example, $\exists x !(x \in t)$ is verified by some models and falsified by others, then

$$
\exists x !(x \in t) \vee !(t \in\{x \mid \sim \exists y !(y \in x) \wedge(x \neq t \vee x \in G)\})
$$

is a minimal $D a b$-theorem. Similarly, if $\vdash_{\text {PFS }} A(t)$ and $t$ is an unclean nonmember of $\{x \mid A(x)\}$ in some but not all PFS-models, then

$$
\begin{aligned}
& (t \notin\{x \mid A(x)\} \wedge \sim \neg A(t)) \vee !(\{x \mid A(x)\} \in\{x \mid \\
& \quad(\forall y(y \in x \Leftrightarrow A(y)) \wedge(t \in x \leftrightarrow A(t))) \wedge(x \neq\{x \mid A(x)\} \vee x \in G)\})
\end{aligned}
$$

is a minimal Dab-theorem. This sixth and seventh road are obtained by the malleability discussed in Section 4. Deciphering (20): either $t$ is an inconsistent set or $t$ is a member of the set of consistent sets of which $t$ is a non-member. Deciphering (21) is left to the reader. The role played by Curry-like sets in the previous paragraphs is played here by (what I shall call) funny normality sets; they comprise normal versions of a certain set as a member, but are inconsistent otherwise. No self-reference or circular reference or similar occurs in (20) or (21). So the vanishing of the adaptive gain is not an effect of odd forms of reference.

The overall conclusion is that $\mathbf{C L u N s}^{m}$ and $\mathbf{C L u N s} \mathbf{s}_{\triangleright}^{m}$ do not define interesting AFS, but AFS that reduce to PFS. This is not a deadlock, however. Ways out have been known for a long time.

\section{Some Adaptive Fregean Set Theories}

Define a sequence $\Omega_{1}, \Omega_{2}, \ldots$ such that (i) $\Omega_{i} \subset \Omega_{i+1}$ for all $i$ and (ii) $\Omega_{1} \cup \Omega_{2} \cup$ $\ldots=\Omega$, where $\Omega$ is as in (9). Let the adaptive logics $\mathbf{C L u N s}_{\triangleright 1}^{m}, \mathbf{C L u N s}_{\triangleright 2}^{m}, \ldots$ be exactly like $\mathbf{C L u N s} \mathbf{s}_{\triangleright}^{m}$ except that the set of abnormalities of $\mathbf{C L u N s} \mathbf{s}_{\triangleright i}^{m}$ is $\Omega_{i}$. The AFS is the sequential superposition:

$$
\ldots \mathrm{Cn}_{\mathbf{C L u N s} s_{\triangleright 3}^{m}}\left(\mathrm{Cn}_{\mathbf{C L u N s}}^{m}{ }_{\triangleright 2}^{m}\left(\mathrm{Cn}_{\mathbf{C L u N s}}{ }_{\triangleright 1}^{m}(\{\mathrm{Abs}, \mathrm{Ext}\})\right)\right) \ldots
$$

There are several straightforward ways to write proof stages of a so defined AFS as sequences rather than as sets of sequences. Below, I consider a few AFS, illustrating some possibilities offered by sequential superpositions. 
As many sets of abnormalities are around, I shall use such expressions as

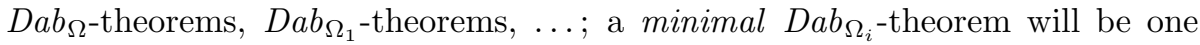
that is minimal among the $D a b_{\Omega_{i}}$-theorems. Note that, for example, $D a b_{\Omega_{3}}$ theorems are CLuNs-derivable from $\mathbf{C n}_{\mathbf{C L u N s}}{ }_{\triangleright 2}^{m}\left(\mathrm{Cn}_{\mathbf{C L u N s}} \mathbf{L u}_{\triangleright 1}^{m}(\{\mathrm{Abs}, \mathrm{Ext}\})\right)$. Such expressions and statements will have a contextual meaning, dependent on the specific sequential superposition of adaptive logics under discussion.

A formula in PNF (prenex normal form) starts with a possibly empty sequence of quantifiers followed by a $\wedge-\vee$-function of elements of the form $\nu t_{1} \xi t_{2}$ in which $\nu$ is nothing or a string from $\{\neg, \sim, \sim \neg, \sim \sim, \sim \sim \neg\}^{29}$ and $\xi \in\{=, \in\}$. $D$ is a PNF of $A$ iff $D$ is in PNF and $\vdash_{\text {CLuNs }} \forall(D \leftrightarrow A)$.

A partition $\left\langle\mathcal{T}_{0}, \mathcal{T}_{1}, \ldots\right\rangle$ of $\mathcal{T}$ will be defined. If $t \in \mathcal{T}_{i}$, then $i$ is said to be the degree of $t$. First, $\mathcal{V} \subseteq \mathcal{T}_{0}$. For abstracting terms, define $\{x \mid A\} \in T_{n}$ iff $n$ is the smallest number such that, for a PNF $D$ of $A, n$ is the sum of (i) the number of occurrences of $\vee$ and $\wedge$ within $D$ and (ii) the maximum of the degrees of the abstracting terms occurring in $D .{ }^{30}$ Some examples: $x \in \mathcal{T}_{0}, \emptyset, U \in \mathcal{T}_{0}$ (because $\vdash_{\mathrm{CLuNs}} \perp \leftrightarrow \exists y \sim y=y$ and $\left.\vdash_{\mathrm{CLuNs}} \top \leftrightarrow \forall y \sim \sim y=y\right),\{\emptyset\},\{\{\emptyset\}\}, \ldots \in \mathcal{T}_{0}$, $R, \bar{\emptyset}, C_{[\perp]}, C_{[\top]} \in \mathcal{T}_{0}, R \cup \emptyset \in \mathcal{T}_{1}$ (because there is one $\vee$ and no $\wedge$ in $x \in R \vee x \in \emptyset$ and $\left.R, \emptyset \in \mathcal{T}_{0}\right),\{\emptyset\} \cup\{\{\emptyset\}\} \in \mathcal{T}_{1}, G \in \mathcal{T}_{1}, C_{[x \in R]} \in \mathcal{T}_{1}, C_{[x \in R]} \cup R \in \mathcal{T}_{2}$ (because there is one $\vee$ and no $\wedge$ in $x \in C_{[x \in R]} \vee x \in R$ and 1 is the maximal degree of $C_{[x \in R]}$ and $\left.R\right), C_{[x \in G]} \in \mathcal{T}_{2},\{x \mid \sim \exists y !(y \in x) \wedge(x \neq \emptyset \vee x \in G)\} \in \mathcal{T}_{4}$, $\{x \mid(\forall y(y \in x \Longleftrightarrow A(y)) \wedge(t \in x \leftrightarrow A(t))) \wedge(x \neq\{x \mid A(x)\} \vee x \in G)\} \in \mathcal{T}_{d}$ where $d$ is either 10 or the degree of $\{x \mid A(x)\}$ plus 9 , whatever is larger, etc.

Define a sequence $\left\langle\Xi_{0}, \Xi_{1}, \ldots\right\rangle$ of subsets of $\Omega$ in terms of the partition of $\mathcal{T}$ - compare to $(9)$ to see the relation between $\Omega$ and the $\Xi_{i} \cdot{ }^{31}$

$$
\begin{aligned}
\Xi_{i}=\left\{\mathrm{Q} !\left(t_{1}=t_{2}\right) \mid t_{1}, t_{2} \in \mathcal{T}_{0} \cup \ldots \cup \mathcal{T}_{i}\right\} \cup \\
\left\{\mathrm{Q} !\left(t_{1} \in t_{2}\right) \mid t_{2} \in \mathcal{T}_{0} \cup \ldots \cup \mathcal{T}_{i}\right\} \cup \\
\left\{\mathrm{Q}(t \in\{y \mid A(y)\} \wedge \sim A(t)) \mid\{y \mid A(y)\} \in \mathcal{T}_{0} \cup \ldots \cup \mathcal{T}_{i}\right\} \cup \\
\quad\left\{\mathrm{Q}(t \notin\{y \mid A(y)\} \wedge \sim \neg A(t)) \mid\{y \mid A(y)\} \in \mathcal{T}_{0} \cup \ldots \cup \mathcal{T}_{i}\right\}
\end{aligned}
$$

Finally, define the sequence $\left\langle\Omega_{1}, \Omega_{2}, \ldots\right\rangle$ by positing $\Omega_{i+1}=\Xi_{i}$. Let $\mathbf{A F S}_{1}$ be the sequential superposition (22), each $\mathbf{C L u N s}_{\triangleright i}^{m}$ being defined by the lower limit logic CLuNs, the set of these $\Omega_{i}$ and the Minimal Abnormality strategy.

The proof that there is an adaptive gain in $\mathbf{A F S}_{1}$ may have the same complexity as a proof that $\mathbf{Z F C}$ is consistent. So this is beyond our reach. Yet, one may establish positive properties of $\mathbf{A F S}_{1}$, for example that it is non-trivial by Theorem 7 and that no know mechanism prevents an adaptive gain, see Theorem 11.

Claim 8 By present lights, there are $A \in \Omega_{1}$ such that $A \notin \Delta$ whenever $D a b_{\Omega_{1}}(\Delta)$ is a minimal Dab $\Omega_{\Omega_{1}}$-theorem of PFS.

The expression by present lights refers to the state of the art and invites the reader to show the author wrong. As for classical theories, relying on similar

\footnotetext{
${ }^{29}$ The table in Section 3 clarifies the matter. Every formula has one or more PNF. Obtaining them is straightforward in view of the CLuNs-semantics; the only unusual step is that $A \supset B$ is eliminated in favour of $\sim A \vee B$, which contains a defined symbol.

${ }^{30}$ That the touchstones of two abstracting terms have the same PNF entails that the terms have the same degree, not that they are identical in all PFS-models.

${ }^{31}$ For expressions of the form $t_{1} \in t_{2}$ only the degree of $t_{2}$ is relevant for determining the $\Xi_{i}$ to which the corresponding abnormality belongs.
} 
claims is unavoidable; $c f$. by present lights PA is consistent. There are arguments in support of the claim. (i) Sets that behave abnormally in PFS are proven to behave so by pretty simple proofs: that $R$ and $Z$ are inconsistent, that $R^{*}$ is unclean with respect to membership, etc. (ii) Nothing suggests that $\emptyset$ or $U$ need be inconsistent and nothing suggests that those sets can only be consistent if other sets are inconsistent; similarly for $\{t\}, \widetilde{t}, \oplus t$ and many other set forming operations. (iii) Nothing suggests that $R$ would be unclean or that $\emptyset$ would be an unclean member of $R^{*}$ or that cleanness for those cases can only be obtained at the expense of having more uncleanness in other sets; similarly for $t_{1}-t_{2}$ or $t_{1} \cup t_{2}$ and other set forming operations. (iv) Nothing suggests that sets that are consistent in the small models from Section 5 would become inconsistent when the domain is extended with consistent sets. (v) If the aforementioned sets would be inconsistent, respectively unclean, one wonders why the sets and set forming operations that also occur in $\mathbf{Z F}$ would behave classically there. That the $\mathbf{A F S}_{1}$-counterpart $t$ of a $\mathbf{Z F}$-set has inconsistent and unclean members or non-members does not cause $t$ to be inconsistent or unclean.

Claim 9 By present lights, $\exists x \exists y !(x=y)$ is not a disjunct of a minimal Dab $b_{\Omega_{1}}$ theorem of PFS.

That is: in minimally abnormal $\mathbf{C L u N s} \mathbf{s}_{\triangleright 1}^{m}$-models of $\{\mathrm{Abs}, \mathrm{Ext}\}$ no two sets are identical as well as different. Apart from specifications of reasons for Claim 8, a particular reason to believe Claim 9 is that all models mentioned in Section 5 are consistent with respect to identity or have a variant that only differs from the original model in that identity is consistent in it. Note that the theoremhood of the third formula in Lemma 10 states that identity is classical in $\mathbf{A F S}_{1}$. This drastically strengthens the way in which Desideratum 2 is fulfilled.

Lemma 10 If Claim 9 holds true, then $\forall x \forall y \sim(x=y \wedge x \neq y), \forall x \sim x \neq x$ and $\forall x \forall y(\neg x=y \leftrightarrow \sim x=y)$ are $\mathbf{A F S}_{1}$-theorems.

Proof. The classical negation of $\exists x \exists y !(x=y)$ is CLuNs-equivalent to $\forall x \forall y \sim(x=$ $y \wedge x \neq y$ ); from this together with the PFS-theorem $\forall x x=x$ follows $\forall x \sim x \neq$ $x$; two conjuncts of $\forall x \forall y(\neg x=y \leftrightarrow \sim x=y)$ are CLuNs-theorems, the two other are derivable on the condition $\exists x \exists y$ ! $(x=y)$.

Theorem 11 If Claim 9 is true, the seven roads from Section 7 do not establish that every $\Omega_{1}$-abnormality is a disjunct of a minimal Dab ${\Omega_{1}}_{1}$-theorem.

Proof outline. Suppose that Claim 9 is true and consider line $\mathrm{i}+1$, where $A \vee$ $D a b_{\Omega_{1}}(\Delta)$ is derived, of each of the seven roads. I shall show that, for some $A$, none of the seven roads establishes that their last line, $\mathrm{i}+3$ or $\mathrm{i}+4$, is a minimal $D a b_{\Omega_{1}}$-formula. This is obvious for roads 3 and 5-7: the constructed sets are not in $\mathcal{T}_{0}$.

Let $A$ of line $\mathrm{i}+1$ be one of the three formulas mentioned in Lemma 10 or an instance of them or another weakening of them and let $\operatorname{Dab}(\Delta)$ be $\exists x \exists y !(x=y)$. For each of these $A$, neither $C_{[\neg A]}$ nor $C_{[\sim A]}$ belongs to $\mathcal{T}_{0}$; even $\sim x \in x \vee \sim(t \neq$ $t$ ) belongs, for all $t$, to a $\mathcal{T}_{i}$ with $i \geq 1$. So roads 1,2 and 4 do not establish that their last line, $\mathrm{i}+3$ or $\mathrm{i}+4$, is a minimal $\operatorname{Dab}_{\Omega_{1}}$-formula.

The proof for the $K_{[\neg A]}$ and $K_{[\sim A]}$ variants proceeds similarly. 
It may also be shown that the seven roads do not prevent normality results for sets and for the membership relation. This claim depends even more on present lights and requires a lengthy proof. I shall retain the results for a subsequent paper that studies theorems of one or more AFS. Salient normality statements include: (a) $t$ is consistent: $\sim \exists x !(x \in t)$; (b) $t_{1}$ is a consistent member or non-member of $t_{2}: \sim !\left(t_{1} \in t_{2}\right) ;(\mathrm{c})\{x \mid A(x)\}$ is clean with respect to membership, respectively non-membership: $\forall x(x \in\{x \mid A(x)\} \supset A(x))$, respectively $\forall x(x \notin\{x \mid A(x)\} \supset \neg A(x))$; (d) $t$ is either not a member or a clean member, respectively not a non-member or a clean non-member, of $\{x \mid A(x)\}$ : $t \in\{x \mid A(x)\} \supset A(t)$, respectively $t \notin\{x \mid A(x)\} \supset \neg A(t)$; (e) variants in quantifiers of (a) and (c) - replace $\exists$ by $\forall$ and vice versa. Each of these formulas $B$ is derivable on a condition $\{D\}$ such that $\vdash_{\text {CLuNs }} \sim B \leftrightarrow D$ (as well as on some other conditions).

By present lights $\mathbf{A F S}_{1}$ is an adaptive Fregean set theory that heavily extends PFS and may be expected to offer a sensible analysis of the Fregean notion of a set. The theory $\mathbf{A F S}_{1}$ is provably non-trivial, handles the known inconsistent sets in a decent way - as consistently as possible, among other things - and, for all we presently know, handles as consistent sets that may be consistent. The latter includes the collections of members and the collections of non-members, $\oplus(t)$ and $\ominus(t)$, of even inconsistent $t$ - there are some exceptions as is discussed below. Also, sets that may be clean are handled as clean by $\mathbf{A F S}_{1}$. This includes the collections of clean members and clean non-members, $\boxplus(t)$ and $\boxminus(t)$, of necessarily unclean $t$-here too some exceptions are discussed below.

Slightly reordering the abnormalities offers new perspectives. Let $\mathbf{A F S}$ be like $\mathbf{A F S}_{1}$ except that the sequence of $\Omega_{i}$ is defined as follows:

$$
\begin{aligned}
\Omega_{1} & =\left\{\mathrm{Q} !\left(t_{1}=t_{2}\right) \mid t_{1}, t_{2} \in \mathcal{T}\right\} \\
\Omega_{2} & =\Omega_{1} \cup\left\{\mathrm{Q} !\left(t_{n+1} \in\left\{t_{1}, \ldots, t_{n}\right\}\right) \mid n \geq 1 ; t_{1}, \ldots, t_{n+1} \in \mathcal{T}\right\} \\
\Omega_{3} & =\Omega_{2} \cup\left\{\mathrm{Q} !\left(t_{1} \in \widetilde{t_{2}}\right), \mathrm{Q} !\left(t_{1} \in \oplus\left(t_{2}\right)\right), \mathrm{Q} !\left(t_{1} \in \ominus\left(t_{2}\right)\right), \mid t_{1}, t_{2} \in \mathcal{T}\right\} \\
\Omega_{i+4} & =\Omega_{i+3} \cup \Xi_{i} \quad(i \in \mathbb{N})
\end{aligned}
$$

Three sets of abnormalities were inserted before $\Xi_{1}$. All differences I found count in favour of $\mathbf{A F S}_{2}$.

In the present $\mathbf{C L u N s} \mathbf{s}_{\triangleright 1}^{m}$-proof, the one with the present choice for $\Omega_{1}$, $\forall x \forall y(\sim x=y \vee \sim x \neq y)$ is derivable on the condition $\{\exists x \exists y !(x=y)\}$. The line at which it is so derived will be unmarked in all extensions of the proof because there are PFS-models in which $v^{I}(=)=\emptyset$. Several small such models were mentioned in Section 5, but it is not difficult to show that, if $M$ is a PFS-model and the assignment of $M^{\prime}$ is exactly like that of $M$ except in that the members of $v^{I}(=)$ in $M$ are members of $v^{T}(=)$ in $M^{\prime}$, then $M^{\prime}$ is a PFS-model-see also Theorem 4. The difference with $\mathbf{A F S}$ is that the proof of Lemma 10 requires Claim 9, which relies on present lights, whereas no such reliance is needed in $\mathbf{A F S}{ }_{2}{ }^{32}$

The $\mathbf{C L u N s} \mathbf{s}_{\triangleright 2}^{m}$-consequence set also offers simple and dependable new theorems: $\forall x \sim !\left(x \in\left\{t_{1}, \ldots, t_{n}\right\}\right)$ is derivable on the condition $\exists x !\left(x=t_{1}\right) \vee \ldots \vee$ $\exists x !\left(x=t_{n}\right)$ and neither disjunct can occur in a minimal $D a b_{\Omega_{2}}$-theorem because, for all $t, \sim \exists x !(x=t)$ is a member of the $\mathbf{C L u N s} \mathbf{s}_{\triangleright 1}^{m}$-consequence set. ${ }^{33}$

\footnotetext{
${ }^{32}$ If the reliance were mistaken in $\mathbf{A F S}{ }_{1}$, the effect in $\mathbf{A F S} \mathbf{S}_{2}$ might be that the membership relation would be less normal than expected.

${ }^{33}$ In view of the sequence of $\Xi_{i}$, it is very likely that $\sim A$ is an $\mathbf{A F S}_{1}$-theorem for any $A$
} 
Minimizing the abnormalities of $\Omega_{3}-\Omega_{2}$ at once minimizes uncleanness. The effect of the present $\Omega_{3}$ is that the sets defined by those operators are consistent as well as clean for nearly all $t_{2}$ - the exceptions will be commented upon. The other involved sets may be consistent and clean and whether they are is not influenced by minimizing abnormalities in $\Omega_{2}$.

What is the effect of the seven roads from Section 7 on $\mathbf{A F S}_{2}$ given its ordering of the $\Omega_{i}$ ? In view of the present $\Omega_{3}$, the theorems of $\mathbf{A F S} \mathbf{S}_{2}$ differ from those of $\mathbf{A} \mathbf{F S}_{1}$, but the adaptive gain is increased rather than diminished by $\mathbf{A F S}_{2}$. The sensitive point is whether $\tilde{t} \in t, \oplus(t) \in t$ and $\ominus(t) \in t$. For many $t$, the sets $\widetilde{t}, \oplus(t)$ and $\ominus(t)$ are as we hoped them to be in both $\mathbf{A F S} \mathbf{S}_{1}$ and $\mathbf{A F} \mathbf{F S}_{2}$. For example, for most $t$, it holds that $\forall x(x \in \oplus(t) \leftrightarrow x \in t)$. This holds even for $Z \in Z$, which is verified by some PFS-models and not by others, and similarly for $\oplus(Z) \in Z$ and $\oplus(Z) \in \oplus(Z)$.

Yet, consider $\oplus\left(R^{*}\right)$. Two relevant instances of Abs give one $\sim \oplus\left(R^{*}\right) \in$ $\oplus\left(R^{*}\right) \supset \oplus\left(R^{*}\right) \in \oplus\left(R^{*}\right)$. So $\vdash_{\mathbf{P F S}} \oplus\left(R^{*}\right) \in \oplus\left(R^{*}\right)$; but then $\vdash_{\mathbf{P F S}} \oplus\left(R^{*}\right) \notin$ $R^{*}$ by Abs. The intended meaning of $\oplus\left(R^{*}\right)$ is just as contradictory as that of $R^{*}$ : in order for $\oplus\left(R^{*}\right)$ to be a clean member of $R^{*}$, we need $\sim \oplus\left(R^{*}\right) \in \oplus\left(R^{*}\right)$. So $\oplus\left(R^{*}\right)$ is definitely not a member of the consistent set of members of $R^{*}$. But then contradiction should and does result: Abs gives one $\vdash_{\text {PFS }} \oplus\left(R^{*}\right) \in$ $R^{*} \vee \oplus\left(R^{*}\right) \notin \oplus\left(R^{*}\right)$, whence follows the $D a b_{\Omega}$-theorem

$$
\vdash_{\mathbf{P F S}} !\left(\oplus\left(R^{*}\right) \in R^{*}\right) \vee !\left(\oplus\left(R^{*}\right) \in \oplus\left(R^{*}\right)\right) .
$$

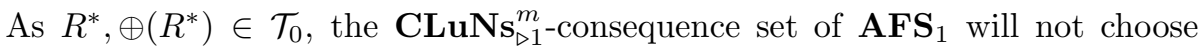
between the disjuncts of $(24)$; neither $\oplus\left(R^{*}\right) \in R^{*}$ nor $\oplus\left(R^{*}\right) \notin \oplus\left(R^{*}\right)$ will

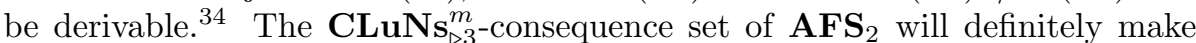
a choice. In view of $(24), !\left(\oplus\left(R^{*}\right) \in R^{*}\right)$ will be derived on the condition $!\left(\oplus\left(R^{*}\right) \in \oplus\left(R^{*}\right)\right)$ because, unlike the former, the latter is a member of the $\Omega_{3}$ of $\mathbf{A F S}_{2}$. It seems highly plausible that the derivation is final: in which way might $!\left(\oplus\left(R^{*}\right) \in \oplus\left(R^{*}\right)\right)$ be a disjunct of a minimal Dab ${ }_{\Omega_{2}}$-formula of AFS $_{2}$ ? Incidentally, $\sim$ ! $\left.\oplus\left(R^{*}\right) \in \oplus\left(R^{*}\right)\right)$ is also derivable on the condition $!\left(\oplus\left(R^{*}\right) \in \oplus\left(R^{*}\right)\right)$. Similar results may be obtained for $\oplus(R), \ominus(Z), \widetilde{\widetilde{R^{*}}}$ and the like.

So $\mathbf{A F S}_{2}$ is preferable over $\mathbf{A F S}_{1}$. As $R^{*}$ has unclean members anyway, including itself, it seems sensible to promote $\oplus\left(R^{*}\right)$, which has a similarly contradictory touchstone, to an unclean member of $R^{*}$. Doing so (i) agrees with the PFS-theorem $\oplus\left(R^{*}\right) \in \oplus\left(R^{*}\right)$ and (ii) arguably turns $\oplus\left(R^{*}\right)$ into a clean and consistent set.

Incidentally, the situation of $\boxplus$ is quite different from that of the operators mentioned in the present $\Omega_{3}$. Both $!\left(\boxplus\left(R^{*}\right) \in \boxplus\left(R^{*}\right)\right)$ and $\boxplus\left(R^{*}\right) \notin R^{*}$ are PFS-theorems, whence $\boxplus\left(R^{*}\right)$ is both inconsistent and unclean (and an unclean member of itself). Of course, $\boxplus(t)$ behaves fine for less paradoxical $t$; even $\boxplus\left(R^{*}\right)$ behaves as it should for 'most' of its members - including $R^{*}$ : both $R^{*} \notin \boxplus\left(R^{*}\right)$ and $R^{*} \in \boxminus\left(R^{*}\right)$ are PFS-theorems. Also, we can state and prove that $\boxplus\left(R^{*}\right)$ is not a clean member of $R^{*}: \sim\left(\boxplus\left(R^{*}\right) \in R^{*} \wedge \sim \boxplus\left(R^{*}\right) \in \boxplus\left(R^{*}\right)\right)$. Yet $\boxplus\left(R^{*}\right)$ is not the clean and consistent set comprising the clean members of $R^{*}$; it is bound to have itself as an unclean member.

\footnotetext{
that is a member of the $\Omega_{2}$ of $\mathbf{A F S}_{2}$. Yet, $\mathbf{A F S _ { 2 }}$ offers certainty.

${ }^{34}$ It is possible that a 'later' $\mathbf{C L u N s} \mathbf{s}_{\triangleright i}^{m}$-consequence set of $\mathbf{A F S} \mathbf{S}_{1}$ introduces further $D a b$ formulas, which eventually lead to a choice. I did not find any.
} 
Consider a third example, $\mathbf{A F S}_{3}$. For some presumed consistent set theories CS, the set of CS-sets, call it $S_{\mathrm{CS}}$, (and the set of CS-classes, call it $C_{\mathrm{CS}}$ ) is definable within AFS and the sentences of CS may be translated into $\mathcal{L}_{S}$ sentences. Further $\mathcal{L}_{S}$-sets may be needed to define $S_{\mathrm{CS}}$ and $C_{\mathrm{CS}}$; let $\mathfrak{H}$ be the metatheoretic set comprising $S_{\mathrm{CS}}, C_{\mathrm{CS}}$ as well as those other $\mathcal{L}_{S^{-}}$-sets.

The set of abnormalities of CS-sets is defined as follows - drop and adjust references to classes as required by the specific CS.

$$
\begin{aligned}
\Omega_{\mathrm{CS}}=\{ & \left.\mathrm{Q} !\left(t_{1}=t_{2}\right) \mid t_{1}, t_{2} \in S_{\mathrm{CS}} \vee t_{1}, t_{2} \in C_{\mathrm{CS}}\right\} \cup \\
& \left\{\mathrm{Q} !\left(t_{1} \in t_{2}\right) \mid t_{1} \in S_{\mathrm{CS}} ; t_{2} \in S_{\mathrm{CS}} \cup C_{\mathrm{CS}}\right\} \cup \\
& \left\{\mathrm{Q}(t \in\{x \mid A(x)\} \wedge \sim A(t)) \mid t \in S_{\mathrm{CS}} ;\{x \mid A(x)\} \in S_{\mathrm{CS}} \cup C_{\mathrm{CS}}\right\} \cup \\
& \left\{\mathrm{Q}(t \notin\{x \mid A(x)\} \wedge \sim \neg A(t)) \mid t \in S_{\mathrm{CS}} ;\{x \mid A(x)\} \in S_{\mathrm{CS}} \cup C_{\mathrm{CS}}\right\}
\end{aligned}
$$

The abnormalities of $\mathfrak{H}$ are defined as follows-adjust for the specific CS.

$$
\begin{aligned}
\Omega_{\mathfrak{H}}=\left\{\mathrm{Q} !\left(t_{1}=t_{2}\right) \mid t_{1}, t_{2} \in \mathfrak{H}\right\} & \cup \\
\left\{\mathrm{Q} !\left(t_{1} \in t_{2}\right) \mid t_{1}, t_{2} \in \mathfrak{H}\right\} & \cup \\
\{\mathrm{Q}(t \in\{x \mid A(x)\} & \wedge \sim A(t)) \mid t,\{x \mid A(x)\} \in \mathfrak{H}\} \cup \\
\{\mathrm{Q}(t \notin\{x \mid A(x)\} & \wedge \sim \neg A(t)) \mid t,\{x \mid A(x)\} \in \mathfrak{H}\}
\end{aligned}
$$

Let $\mathbf{A F S} \mathbf{S}_{3}$ be like $\mathbf{A F S}{ }_{1}$ except that the $\Omega_{i}$ are defined as follows:

$$
\begin{aligned}
\Omega_{1} & =\left\{\mathrm{Q} !\left(t_{1}=t_{2}\right) \mid t_{1}, t_{2} \in \mathcal{T}\right\} \\
\Omega_{2} & =\Omega_{1} \cup \Omega_{\mathfrak{H}} \\
\Omega_{3} & =\Omega_{2} \cup \Omega_{\mathrm{CS}} \\
\Omega_{i+4} & =\Omega_{i+3} \cup \Xi_{i} \quad(i \in \mathbb{N})
\end{aligned}
$$

The first set in the union of the definiens of $\Omega_{\mathrm{CS}}$ and the first set in the union of the definiens of $\Omega_{\mathfrak{H}}$ may obviously be dropped in view of the present $\Omega_{1}$. While $\Omega_{2}$ warrants that the members of $\mathfrak{H}$ are consistent and clean, $\Omega_{3}$ warrants that the members of those members are consistent and clean. The reasons to believe that $\Omega_{2}$ and $\Omega_{3}$ provide those warrants coincide with the reasons to believe that CS is a consistent set theory.

Fact 12 If CS-sets (and CS-classes) form a $\mathbf{A F S}_{3}$-set, then if $\mathrm{CS}$ is indeed consistent, then CS is a consistent and clean subtheory of $\mathbf{A F S}_{3}$.

What I mean by a subtheory is that any CS-theorem $A$ corresponds to an AFS $_{3}$-theorem $t(A)$; in the simplest case $t(A)=B \supset A$ in which $B$ states that all sets mentioned in $A$ are CS-sets.

All AFS require study. One approach reorders the $\Omega_{i}$ to establish final provability as illustrated by $\mathbf{A F S}_{2}$. A second approach aims at showing that many operators (unions, intersections, ...) define sets the cleanness of which has no effect on the normality of other sets except for artificial constructions (Currylike sets, funny normality sets, and presumably more). The operators function as desired if they define sets with a lower degree than the artificial constructions. A third approach capitalizes on presumed consistent set theories CS in view of (i) if $\Gamma \vdash_{\mathbf{C L u N s}} A$, then $\Gamma \vdash_{\mathbf{C L}} A$, (ii) if $\Gamma \vdash_{\mathbf{C L}} A$, then there is a finite $\Delta \subset \Omega$ such that $\Gamma \vdash_{\mathbf{C L u N s}} A \vee \operatorname{Dab}(\Delta)$, and (iii) if $\Gamma \vdash_{\mathbf{C L}} A$, CS is consistent 
and certain (not too exotic) conditions are fulfilled by the AFS, then $\vdash_{\text {AFS }} A$ whenever $\vdash_{\mathrm{AFS}} B$ for all $B \in \Gamma$. This approach need not be restricted to a single CS. A fourth approach introduces one or more $S_{\mathrm{CS}}$ as illustrated by $\mathbf{A F S}_{3}$. New problems may arise, especially concerning the translation functions. Different ways for incorporating such theories may have to be devised. If some presumably consistent first order set theories would resist incorporation, this might reveal a potentially fatal restriction on adaptive Fregean set theories. A fifth approach concerns the study of the set of consistent AFS-sets. This may be interpreted naively, but one may also delineate a consistent set theory within an $\mathbf{A F S}_{i}$ by devising an as large as possible set $S$ the members of which are consistent and have only members of $S$ as members. This provides ways to devise alternative consistent set theories. That it happens within an AFS will definitely have heuristic advantages, but possibly also technical advantages. A sixth approach handles lots of sensible work that goes in the opposite direction. Implicitly, PFS-models were described within a classic set theory - clearly ZFC will do. Each adaptive logic in the sequential superposition (22) selects a set of PFS-models of its premisses and its consequence set comprises the $\mathcal{L}_{S}$-formulas true in all selected models. The dynamic proofs of the adaptive logics and of their sequential superposition can also be described in terms of ZFC. All this suggests that any AFS can be embedded within ZFC and other 'classic' set theories.

Incidentally, if a $\mathbf{C L u N s} \mathbf{s}_{\triangleright i}^{m}$ of a certain $\mathbf{A F S}_{j}$ would destroy the adaptive gain, it reduces to CLuNs. But that is harmless: all $\mathbf{C L u N s} \mathbf{s}_{\triangleright i}^{m}$ in (22) deliver a consequence set that is closed under CLuNs as well as transitive.

\section{In Conclusion}

The main purpose of the present paper was to offer examples of theories that characterize Frege's notion of a set, taking into account that the notion requires inconsistent sets, and to spell out the method for devising such theories. The five desiderata from Section 1 are all fulfilled. Desideratum 4 will be better understood at this point in the paper in terms of $D a b$-formulas. A specific problem for adaptive set theories pertained to the adaptive gain and was adequately solved.

In subsequent paragraphs, I shall first comment on adaptive Fregean set theories and next on two implications for the methodology of adaptive logics.

The idea behind my approach is double. (1) Frege's idea was that $A(t)$ is the support of $t \in\{x \mid A(x)\}$ and $\neg A(t)$ the support of $t \notin\{x \mid A(x)\}$. Yet $R \in R$ and $R \notin R$ are proved, in Frege's original theory and PFS alike, without support, viz. from $R \notin R \supset R \in R$ and $\neg R \notin R \supset R \notin R$. This causes inconsistency. More significant are the separate steps: if only one of them applied, one would obtain set membership without support whence an unclean set. That both apply in the case of $R$ hides the lack of support and blurs the insight. The fundamental problem is uncleanness; inconsistency is a side effect. In this respect $R^{*}$ is significant: $R^{*}$ is not $\sim$-inconsistent in PFS and hence unclean. (2) The second idea pursues the first: the adaptive gain is eaten by abstracting terms that have unsupported members or non-members; such terms cause supported set membership to be involved in $D a b$-formulas of the $\mathbf{C L u N s} \mathbf{s}_{\triangleleft}^{m}$-theory. The insight led to a complicated ordering of subsets of the $\Omega$ from (9). As only the relation between touchstones matters, the present 
$\Xi$-sequence arguably offers a drastic but satisfactory simplification.

Inconsistent sets may be thought of as couples of consistent sets. This may be expressed within $\mathbf{A F S} \mathbf{S}_{2}$ where both members of $\langle\oplus t, \ominus t\rangle$ are arguably consistent sets. In return, for any two consistent sets $t_{1}$ and $t_{2}$, the couple $\left\langle t_{1}, t_{2}\right\rangle$ defines a Fregean set provided $\forall x\left(x \in t_{1} \vee x \in t_{2}\right)$ holds. The phrase may be read as a statement on PFS-models described in terms of any decent set theory, including an AFS, in which case the quantifiers are interpreted with respect to a domain D. It may also be read as an absolute statement in terms of a decent set theory, provided the latter allows for consistent sets $t_{1}$ and $t_{2}$ for which the condition holds.

Some collections of sets were sometimes said too large to be consistent sets. Yet some AFS enable one to handle many of those collections precisely so: $U$, $\widetilde{t}, \oplus(R), \ominus(R), \oplus\left(R^{*}\right)$, and so on. Some such sets, for example $\oplus(Z)$, are contingent on the model but nevertheless consistent in all AFS-models.

The local classical negation plays a significant role within PFS and within the AFS. First, many functions that generate consistent sets in the AFS do so in view of the presence of the classical negation-Section 3 offers many examples of sets that cannot be defined without a classical negation. Next, the classical negation offers a simple means to express that there is a gain: some $t_{1} \neq t_{2}$ have the force of $\sim t_{1}=t_{2}$ and some $t_{1} \notin t_{2}$ the force of $\sim t_{1} \in t_{2} \cdot{ }^{35}$ Third, the classical negation allows one to express that a set $t$ is consistent: $\sim \exists x(x \in t \wedge x \notin$ t). ${ }^{36}$ Fourth, the presence of this classical negation does not cause triviality. ${ }^{37}$

As I extensively explained in Section 4 , the language $\mathcal{L}_{S}$ is such that $\mathbf{C L u N s}$ distinguishes between several implications that coincide according to $\mathbf{C L}$ and the same holds even more for equivalences; so one has to make a choice anyway. Yet, some might think to have 'arguments' for the claim that the equivalence in Abs should be symmetric. They are easily satisfied by moving the asymmetry from the connective to the superposed adaptive logics. Take any of the AFS defined in Section 8. Phrase Abs in terms of the LP-equivalence, $A \Leftrightarrow B$ in the terminology of the present paper, and call the result Abs $\Leftrightarrow$. Renumber the $\Omega_{i}$ to $\Omega_{i+1}$ and define $\Omega_{1}$ as follows.

$\left\{\mathrm{Q}(A(t) \wedge \sim t \in\{y \mid A(y)\}) \mid A \in \mathcal{F}_{S}\right\} \cup\left\{\mathrm{Q}(\neg A(t) \wedge \sim t \notin\{y \mid A(y)\}) \mid A \in \mathcal{F}_{S}\right\}$

The new $\mathbf{A F S}_{i}$ delivers exactly the same consequence set as the old one because, as the old PFS was non-trivial anyway, the new $\mathbf{C L u N s} \mathbf{s}_{\triangleright 1}^{m}$ eliminates all

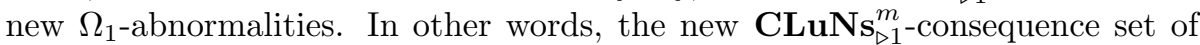
$\{\mathrm{Abs} \Leftrightarrow, \mathrm{Ext}\}$ is identical to the CLuNs-closure of $\{\mathrm{Abs}, \mathrm{Ext}\}$ and it is provable that the consequence set assigned by an adaptive logic $A L$ to a premise set $\Gamma$ is identical to the consequence set assigned by $\mathrm{AL}$ to the closure of $\Gamma$ by the lower limit logic of AL [3, Theorem 15,(3)]. In the present case the AL is the old $\mathbf{C L u N s} s_{\triangleright 1}^{m}$, which is the new $\mathbf{C L u N s} \mathbf{s}_{\triangleright 2}^{m}$. Let us stop the frivolity here and

\footnotetext{
${ }^{35}$ There is no need for a classical negation in order to have the gain: if, for example, $A \wedge \neg A$ can be upheld to be false, $B$ can be derived from $\neg A$ and $A \vee B$.

${ }^{36}$ While $\exists x(x \in t \wedge x \notin t)$ definitely expresses that $t$ is inconsistent, $\neg \exists x(x \in t \wedge x \notin t)$, being a CLuNs-theorem, offers a slightly too easy way to prove the consistency of $t$.

${ }^{37}$ This is realized by having the equivalence of Abs non-detachable in the left-right direction. But why would that decrease the merits of the AFS? Graham Priest [17] propagates, as the foundational tool for building LP-models, a set theory which lacks connectives that are detachable in any respect. Also, I have argued, in tempore non suspecto, that minimally abnormal interpretations of inconsistent classical theories may be obtained by weakening other logical symbols than the classical negation $[1,7]$.
} 
let PFS and the AFS again refer to what they referred to before the present paragraph. ${ }^{38}$

An important point concerns the methodology of adaptive logics in general. When a classical theory $T$, which obviously was intended as consistent, turns out inconsistent, no general purpose trick turns $T$ into an adequate minimally abnormal theory. A suitable adaptive approach is only reached by study and analysis of the relevant domain. The present paper illustrates that clearly: the choice of the implications and equivalences in the axioms, the introduction of abnormalities relating to cleanness, and the need for a sequential superposition and more precisely for one that saves the adaptive gain. Handling inconsistency as here discussed is a methodological matter and, solving standard problems aside, interesting methods are content driven or content guided [19].

The present paper clearly suggests that abnormalities do not concern the structure of models - the domain D and the sets defined over it-but concern the relation between the language and the models. Abnormalities pertain to the assignment, to the way in which the abstracting terms are mapped on the domain - so to the models in the plain sense: $M=\langle\mathrm{D}, v\rangle$. The $\mathbf{C L u N s}^{{ }^{m}}$ models of $\Gamma$ are not those in which certain inconsistent sets are absent. Suppose that $\mathrm{D}=\{l, m, r, u\}$ and that we represent (paraconsistent) sets by the couple of the collection of their members and the collection of their non-members, as in $\langle\{l, m\},\{r, u\}\rangle$. Then what might it even mean that $\langle\{l, m\},\{l, r, u\}\rangle$ does not exist in a model? The CLuNs ${ }^{m}$-models of $\Gamma$ are the CLuNs-models of $\Gamma$ in which the linguistic entities are mapped to sets in such a way that verified inconsistencies are minimized.

Concluding the paper, I hope to have shown that the adaptive study of Frege's notion of a set is not only sensible but also promising. It is even promising for studying the variety of consistent set theories. It is correct that AFS are complex theories $[9,12,13,23]$ whence establishing the final derivability of certain formulas may be difficult and tentative. In return, there is a nontriviality proof - so a proof of -consistency. So even if obtaining a warrant of theoremhood may be demanding for some formulas and may seem hopeless for some others, one knows that the theory one is spelling out cannot possibly turn out a chimera.

\section{References}

[1] Batens, Diderik, 'A survey of inconsistency-adaptive logics', in Diderik Batens, Chris Mortensen, Graham Priest, and Jean Paul Van Bendegem, (eds.), Frontiers of Paraconsistent Logic, Research Studies Press, Baldock, UK, 2000, pp. 49-73.

[2] Batens, Diderik, 'A general characterization of adaptive logics', Logique et Analyse, 173-175 (2001), 45-68. Appeared 2003.

\footnotetext{
${ }^{38}$ Similarly, one may modify Ext to $\forall x \forall y(\forall z(z \in x \leftrightarrow z \in y) \sqsupset x=y)$ and modify $\Omega_{1}$ to $\left\{\mathrm{Q}(A(t) \wedge \sim t \in\{y \mid A(y)\}) \mid A \in \mathcal{F}_{S}\right\} \cup\left\{\mathrm{Q}(\neg A(t) \wedge \sim t \notin\{y \mid A(y)\}) \mid A \in \mathcal{F}_{S}\right\} \cup\{\mathrm{Q}(\forall z(z \in$ $x \leftrightarrow z \in y) \wedge \sim x=y)\}$. Again the new $\mathbf{C L u N s} \mathbf{s}_{\triangleright 1}^{m}$-consequence set of the so modified axioms is identical to the CLuNs-closure of $\{\mathrm{Abs}, \mathrm{Ext}\}$. However, $\leftrightarrow$ cannot be replaced by $\Leftrightarrow$ in Ext; no adaptive logic can repair the too weak sufficient condition.
} 
[3] Batens, Diderik, 'A universal logic approach to adaptive logics', Logica Universalis, 1 (2007), 221-242.

[4] BAtens, Diderik, 'Towards a dialogic interpretation of dynamic proofs', in Cédric Dégremont, Laurent Keiff, and Helge Rückert, (eds.), Dialogues, Logics and Other Strange Things. Essays in Honour of Shahid Rahman, College Publications, London, 2009, pp. 27-51. 558 pp.

[5] Batens, Diderik, 'The consistency of Peano Arithmetic. A defeasible perspective', in Patrick Allo, and Bart Van Kerkhove, (eds.), Modestly Radical or Radically Modest. Festschrift for Jean Paul van Bendegem on the Occasion of His 60th Birthday, vol. 24 (sometimes 22) of Tributes, College Publications, London, 2014, pp. 11-59.

[6] Batens, Diderik, 'Tutorial on inconsistency-adaptive logics', in JeanYves Béziau, Mihir Chakraborty, and Soma Dutta, (eds.), New Directions in Paraconsistent Logic, vol. 152 of Springer Proceedings in Mathematics E Statistics, Springer, 2015, pp. 3-38.

[7] Batens, Diderik, 'Spoiled for choice?', Journal of Logic and Computation, 26 (2016), 1, 65-95. E-published 2013: doi:10.1093/logcom/ext019.

[8] Batens, Diderik, and Kristof De ClercQ, 'A rich paraconsistent extension of full positive logic', Logique et Analyse, 185-188 (2004), 227-257. Appeared 2005.

[9] Batens, Diderik, Kristof De Clercq, Peter Verdée, and Joke Meneus, 'Yes fellows, most human reasoning is complex', Synthese, 166 (2009), 113-131.

[10] Brady, Ross, Universal Logic, CSLI Publications, Stanford, Cal., 2006.

[11] da Costa, Newton C.A., and Elias H. Alves, 'A semantical analysis of the calculi $\mathbf{C}_{n}$ ', Notre Dame Journal of Formal Logic, 18 (1977), 621-630.

[12] Odintsov, Sergei P., and Stanislav O. Speranski, 'On algorithmic properties of propositional inconsistency-adaptive logics', Logic and Logical Philosophy, 21 (2012), 209-228.

[13] Odintsov, Sergei P., and Stanislav O. Speranski, 'Computability issues for adaptive logics in multi-consequence standard format', Studia Logica, 101 (2013), 6, 1237-1262. Doi:10.1007/s11225-013-9531-2.

[14] Priest, Graham, 'Minimally inconsistent LP', Studia Logica, 50 (1991), $321-331$.

[15] Priest, Graham, 'Is arithmetic consistent?', Mind, 103 (1994), 337-349.

[16] Priest, Graham, 'Inconsistent models of arithmetic. Part I: Finite models', Journal of Philosophical Logic, 26 (1997), 223-235.

[17] Priest, Graham, In Contradiction. A Study of the Transconsistent, Oxford University Press, Oxford, 2006. Second expanded edition (first edition 1987). 
[18] Quine, Willard Van Orman, 'On the theory of types', The Journal of Symbolic Logic, 3 (1938), 4, 125-139.

[19] Shapere, Dudley, 'Logic and the philosophical interpretation of science', in Paul Weingartner, (ed.), Alternative Logics. Do sciences need them?, Springer, Berlin, Heidelberg, 2004, pp. 41-54.

[20] Strasser, Christian, Adaptive Logic and Defeasible Reasoning. Applications in Argumentation, Normative Reasoning and Default Reasoning., vol. 38 of Trends in Logic, Springer, 2014.

[21] Van Bendegem, Jean Paul, 'Strict, yet rich finitism', in Z.W. Wolkowski, (ed.), First International Symposium on Gödel's Theorems, World Scientific, Singapore, 1993, pp. 61-79.

[22] Van Bendegem, Jean Paul, 'Strict finitism as a viable alternative in the foundations of mathematics', Logique et Analyse, 145 (1994), 23-40. Appeared 1996.

[23] Verdée, Peter, 'Adaptive logics using the minimal abnormality strategy are $\Pi_{1}^{1}$-complex', Synthese, 167 (2009), 93-104.

[24] Verdée, Peter, 'Non-monotonic set theory as a pragmatic foundation of mathematics', Foundations of Science, 18 (2013), 655-680.

[25] Verdée, Peter, 'Strong, universal and provably non-trivial set theory by means of adaptive logic', Logic Journal of the IGPL, 21 (2013), 1, 108-125.

[26] WeBer, ZACH, 'Extensionality and restriction in naive set theory', Studia Logica, 94 (2010), 87-104.

[27] WeBer, ZACH, 'Transfinite numbers in paraconsistent set theory', Review of Symbolic Logic, 3 (2010), 71-92.

[28] WeBer, ZACH, 'Transfinite cardinals in paraconsistent set theory', Review of Symbolic Logic, 5 (2012), 269-293. 